\title{
Anatomy of the binary black hole recoil: A multipolar analysis
}

\author{
Jeremy D. Schnittman, ${ }^{1}$ Alessandra Buonanno, ${ }^{1}$ James R. van Meter, ${ }^{2}{ }^{3}$ John G. Baker ${ }^{2}$ \\ William D. Boggs ${ }^{4}$ Joan Centrella, ${ }^{2}$ Bernard J. Kelly, ${ }^{2}$ and Sean T. McWilliams ${ }^{4}$ \\ ${ }^{1}$ Maryland Center for Fundamental Physics, Department of Physics, \\ University of Maryland, College Park, Maryland 20742 \\ ${ }^{2}$ Gravitational Astrophysics Laboratory, NASA Goddard Space Flight Center, 8800 Greenbelt Rd., Greenbelt, MD 20771 \\ ${ }^{3}$ Center for Space Science \& Technology, University of Maryland Baltimore County, \\ Physics Department, 1000 Hilltop Circle, Baltimore, MD 21250 \\ ${ }^{4}$ Department of Physics, University of Maryland, College Park, Maryland 20742
}

(Dated: October 26, 2018)

\begin{abstract}
We present a multipolar analysis of the gravitational recoil computed in recent numerical simulations of binary black hole (BH) coalescence, for both unequal masses and non-zero, non-precessing spins. We show that multipole moments up to and including $\ell=4$ are sufficient to accurately reproduce the final recoil velocity (within $\simeq 2 \%$ ) and that only a few dominant modes contribute significantly to it (within $\simeq 5 \%$ ). We describe how the relative amplitudes, and more importantly, the relative phases, of these few modes control the way in which the recoil builds up throughout the inspiral, merger, and ringdown phases. We also find that the numerical results can be reproduced by an "effective Newtonian" formula for the multipole moments obtained by replacing the radial separation in the Newtonian formulae with an effective radius computed from the numerical data. Beyond the merger, the numerical results are reproduced by a superposition of three Kerr quasinormal modes (QNMs). Analytic formulae, obtained by expressing the multipole moments in terms of the fundamental QNMs of a Kerr BH, are able to explain the onset and amount of "anti-kick" for each of the simulations. Lastly, we apply this multipolar analysis to help explain the remarkable difference between the amplitudes of planar and non-planar kicks for equal-mass spinning black holes.
\end{abstract}

PACS numbers: 04.25.Dm, 04.30.Db, 04.70.Bw, 04.25.Nx, 04.30.-w

\section{INTRODUCTION}

After the recent breakthrough in numerical relativity (NR) [1, 2, 3], a number of different groups are now able to evolve binary black holes $(\mathrm{BHs})$ through merger [4, 5, 6]. Recently, a great deal of effort has been directed towards the computation of the recoil velocity of the final BH $7,8,8,10,11,12,13,14,16$. The fundamental cause of this recoil is a net linear momentum flux in the gravitational radiation, due to some asymmetry in the system [19, 20, 21, 22, 23], typically unequal masses or spins in the case of $\mathrm{BH}$ binaries. The recoil has great astrophysical importance because it can affect the growth of supermassive black holes (SMBHs) in the early universe 24, 25, 26, 27]. In those scenarios dark-matter haloes grow through hierarchical mergers. The SMBHs at the centers of such haloes are expected to merge unless they have been kicked out of the gravitational potential well because the recoil velocity gained in a prior merger is larger than the halo's escape velocity.

Other astrophysical implications include the displacement of the $\mathrm{SMBH}$, along with its gaseous accretion disk, forming an "off-center" quasar [28]. These quasars might also have emission lines highly red- or blue-shifted relative to the host galaxy due to the Doppler shift of the recoil velocity 29]. Additionally, these displaced SMBHs could in turn displace a significant amount of stellar mass from the galactic nucleus as they sink back to the center via dynamical friction, forming a depleted core of missing mass on the order of twice the SMBH mass [25, 30, 31].
Numerical simulations have now been used to compute recoil velocities for non-spinning unequal-mass BH binary systems [7, 8, 9] in the range $m_{2} / m_{1}=(1 \cdots 4)$, where $m_{1}$ and $m_{2}$ are the individual $\mathrm{BH}$ masses; for spinning, non-precessing binary BHs [10, 11, 12], and also for precessing BHs with both equal [13, 14] as well as unequal masses [16]. Quite interestingly, there exist initial spin configurations for which the recoil velocity can be quite large, e.g., $\gtrsim 3000 \mathrm{~km} / \mathrm{sec}$ [13, 14, 15, 16]. However, it is not yet clear whether those very large recoil velocities are astrophysically likely [26, 33, 34, 35]. So far, due to limited computational resources, the numerical simulations have explored a rather small portion of the total parameter space.

Analytic calculations, based on the post-Newtonian (PN) expansion of Einstein's field equations 36 and PNresummation techniques [37, 38, 39, 40, 41, 42], have made predictions for the recoil velocity [43, 44, 45, 46, 47] before the NR breakthrough. Since the majority of the linear momentum flux is emitted during the merger and ringdown (RD) phases, it is difficult to make definitive predictions for the recoil using only analytic methods. These methods need to be somehow calibrated to the NR results, so that they can be accurately extended during the transition from inspiral to RD. So far, in the nonspinning case, the PN model [46] has provided results consistent with NR all along the adiabatic inspiral; the effective-one-body (EOB) model [37, 38, 40] can reproduce the total recoil, including the contribution from the $\mathrm{RD}$ phase, but with large uncertainties [47]. In Ref. [48], 
perturbative calculations that make use of the so-called close-limit approximation [49] have been used to predict the recoil for unequal-mass binary BHs moving on circular and eccentric orbits. More recently, Ref. [32] provided the first estimates of the distribution of recoil velocities from spinning $\mathrm{BH}$ mergers using the EOB model, calibrated to the NR results.

In this paper we present a diagnostic of the physics of the recoil, trying to understand how it accumulates during the inspiral, merger, and RD phases. The majority of the analysis is based on several numerical simulations of non-spinning, unequal-mass binary systems, as well as spinning, non-precessing binary systems obtained by the Goddard numerical relativity group. What we learn in this study will be used in a forthcoming paper to improve the PN analytic models 32, 46, 47], so that they can be used to interpolate between NR results, efficiently and accurately covering the entire parameter space.

We frame our understanding using the multipolar formalism originally laid out by Thorne [50, 51, 52, 53, 54 ]. We work out which multipole moments contribute most significantly to the recoil. We employ analytic, but leading order, formulae for the linear momentum flux during the inspiral phase, and express the multipole moments in terms of a linear superposition of quasi-normal modes (QNMs) during the RD phase [55]. These analysis tools help us understand why for some binary mass and spin configurations the so-called "anti-kick" is larger than in other cases. By anti-kick, we mean that the recoil velocity reaches a maximum value before decreasing to a final, smaller velocity asymptotically. As shown in Ref. [12], even a relatively small range of binary parameters can give rise to a large variety of anti-kick magnitudes (and even the complete lack of an anti-kick in some cases).

An example of this multipole analysis is shown in Fig. 1. which plots the recoil velocity as a function of time (black curve), along with the separate contributions from the mass-quadrupole-mass-octupole (red), mass-quadrupole- current-quadrupole (blue), and massquadrupole-mass-hexadecapole (green) moments. This plot corresponds to a non-spinning system with mass ratio of 1:2. Note in particular how the modes add both constructively and destructively to give the total recoil. For the non-spinning, unequal-mass systems, the kick and anti-kick are dominated by the mass-quadrupolemass-octupole modes, but also receive significant contributions from the other mode-pairs. For all of the simulations presented in this paper, we scale the time axis around $t_{\text {peak }}$, the time at which the mass quadrupole mode reaches a maximum, closely corresponding to the peak in gravitational wave power, as well as the time that a single horizon is formed and the ringdown phase begins.

This paper is organized as follow. In Sec. II after introducing our definitions and notations, we review the binary parameters used in the numerical simulations and examine the main features of the numerical runs. In Sec. III we discuss the multipolar expansion of the linear momentum, angular momentum and energy fluxes given

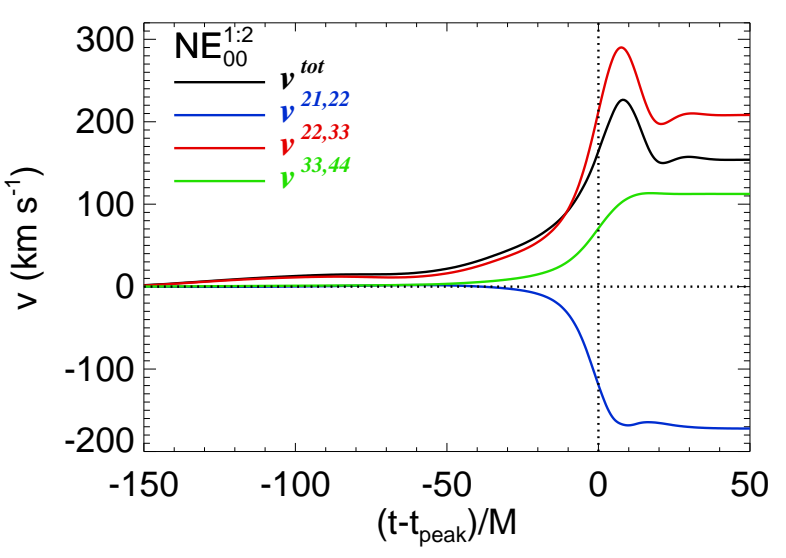

FIG. 1: The recoil velocity as a function of time for a binary $\mathrm{BH}$ system with mass ratio 1:2 and no spins. The total recoil is plotted in black, along with the contributions from different mode pairs, described below in Sec. III We denote by $t_{\text {peak }}$ the time at which the multipole $I^{22}$ reaches its maximum (see Section III).

in terms of the symmetric trace-free radiative mass and current moments, and show how to compute those fluxes from the multipole decomposition of the Weyl scalar $\Psi_{4}$. In Sec. IV] we analyse the multipole content of the numerical waveforms during the inspiral and ringdown phases. In Sec. V we show that, by properly normalizing the binary radial separation, the multipole moments computed at leading order in an expansion in $1 / c$ can approximate quite well the numerical results. Moreover, a superposition of three QNMs matches the RD phase. In Sec. VI we apply the tools developed in the previous sections to understand, using analytic expressions, how the kick builds up during the inspiral, merger, and ringdown phases. We also apply these methods to help explain the large difference between planar and non-planar kicks from equal-mass spinning BHs [10, 13, 16]. Finally, Sec. VII contains a brief discussion of our main results and future research directions. In Appendix $\mathrm{A}$ we discuss recent results for mass ratio $1: 4$.

\section{NUMERICAL SIMULATIONS}

In this section we introduce our definitions and notation, and review the main features of the numerical simulations. Throughout the paper, we adopt geometrical units with $G=c=1$ (unless otherwise specified) and metric signature $(-1,1,1,1)$.

\section{A. Definitions and conventions}

Our complex null tetrad is defined using the time-like unit vector normal to a given hypersurface $\hat{\tau}$, the radial 
unit vector $\hat{r}$, and ingoing $(\vec{\ell})$ and outgoing $(\vec{n})$ null vectors as

$$
\begin{aligned}
\vec{\ell} & \equiv \frac{1}{\sqrt{2}}(\hat{\tau}+\hat{r}), \\
\vec{n} & \equiv \frac{1}{\sqrt{2}}(\hat{\tau}-\hat{r}) .
\end{aligned}
$$

We define the complex null vectors $\vec{m}$ and $\vec{m}^{*}$ by

$$
\begin{aligned}
\vec{m} & \equiv \frac{1}{\sqrt{2}}(\hat{\theta}+i \hat{\varphi}), \\
\vec{m}^{*} & \equiv \frac{1}{\sqrt{2}}(\hat{\theta}-i \hat{\varphi}),
\end{aligned}
$$

with the standard spherical metric at infinity $d s^{2}=$ $-d \tau^{2}+d r^{2}+r^{2}\left(d \theta^{2}+\sin ^{2} \theta d \varphi^{2}\right)$. The orthogonality relations of this tetrad are then

$$
\begin{aligned}
\vec{\ell} \cdot \vec{\ell} & =\vec{n} \cdot \vec{n}=\vec{m} \cdot \vec{m}=\vec{m}^{*} \cdot \vec{m}^{*}=0, \\
\vec{\ell} \cdot \vec{n} & =-\vec{m} \cdot \vec{m}^{*}=-1, \\
\vec{\ell} \cdot \vec{m} & =\vec{\ell} \cdot \vec{m}^{*}=\vec{n} \cdot \vec{m}=\vec{n} \cdot \vec{m}^{*}=0 .
\end{aligned}
$$

In terms of this tetrad, the complex Weyl scalar $\Psi_{4}$ is given by

$$
\Psi_{4} \equiv C_{a b c d} n^{a}\left(m^{b}\right)^{*} n^{c}\left(m^{d}\right)^{*}
$$

where $C_{a b c d}$ is the Weyl tensor and * denotes complex conjugation.

To relate $\Psi_{4}$ to the gravitational waves (GWs), we note that in the transverse-traceless (TT) gauge (see Chap. 35 in Ref. [17]),

$$
\begin{aligned}
\frac{1}{4}\left(\ddot{h}_{\hat{\theta} \hat{\theta}}^{T T}-\ddot{h}_{\hat{\varphi} \hat{\varphi}}^{T T}\right) & =-R_{\hat{\tau} \hat{\theta} \hat{\tau} \hat{\theta}}=-R_{\hat{\tau} \hat{\varphi} \hat{r} \hat{\varphi}}=-R_{\hat{r} \hat{\theta} \hat{r} \hat{\theta}} \\
& =R_{\hat{\tau} \hat{\varphi} \hat{\tau} \hat{\varphi}}=R_{\hat{\tau} \hat{\theta} \hat{r} \hat{\theta}}=R_{\hat{r} \hat{\varphi} \hat{r} \hat{\varphi}} \\
\frac{1}{2} \ddot{h}_{\hat{\theta} \hat{\varphi}}^{T T}=-R_{\hat{\tau} \hat{\theta} \hat{\tau} \hat{\varphi}} & =-R_{\hat{r} \hat{\theta} \hat{r} \hat{\varphi}}=R_{\hat{\tau} \hat{\theta} \hat{r} \hat{\varphi}}=R_{\hat{r} \hat{\theta} \hat{\tau} \hat{\varphi}}
\end{aligned}
$$

Following usual convention, we take the $h_{+}$and $h_{\times}$polarizations of the GW to be given by

$$
\begin{aligned}
& \ddot{h}_{+}=\frac{1}{2}\left(\ddot{h}_{\hat{\theta} \hat{\theta}}^{T T}-\ddot{h}_{\hat{\varphi} \hat{\varphi}}^{T T}\right), \\
& \ddot{h}_{\times}=\ddot{h}_{\hat{\theta} \hat{\varphi}}^{T T} .
\end{aligned}
$$

Since the Riemann and Weyl tensors coincide in vacuum regions of the spacetime $\left(R_{a b c d}=C_{a b c d}\right)$, we find by combining the above equations:

$$
\Psi_{4}=-\left(\ddot{h}_{+}-i \ddot{h}_{\times}\right)
$$

Note that this expression for $\Psi_{4}$ is tetrad-dependent. Here we assume the tetrad given in Ref. [18], Eqs. (5.6). It is also common for $\Psi_{4}$ to be scaled according to an asymptotically Kinnersley tetrad (Ref. [18], Eqs. (5.9)) which introduces a factor of 2 as in Ref. [68].

It is most convenient to deal with $\Psi_{4}$ in terms of its harmonic decomposition. Given the definition of $\Psi_{4}$ in Eq. (41) and the fact that $\vec{m}^{*}$ carries a spin-weight of -1 , it is appropriate to decompose $\Psi_{4}$ in terms of spin-weight -2 spherical harmonics ${ }_{2} Y_{\ell m}(\theta, \varphi)$ [56]. There is some freedom in the definition of the spin-weighted spherical harmonics. Here, we define them as a linear combination of the scalar spherical harmonics $Y_{\ell m}$ and $Y_{(\ell-1) m}$, as in Ref. [57]:

$$
{ }_{ \pm 2} Y_{\ell m}(\theta, \varphi)=\left[\frac{(\ell-2) !}{(\ell+2) !}\right]^{1 / 2}\left[\alpha_{(\ell m)}^{ \pm}(\theta) Y_{\ell m}(\theta, \varphi)+\beta_{(\ell m)}^{ \pm}(\theta) Y_{(\ell-1) m}(\theta, \varphi)\right]
$$

for $\ell \geq 2$ and $|m| \leq \ell$, and with the functional coefficients

$$
\begin{aligned}
& \alpha_{(\ell m)}^{ \pm}(\theta)=\frac{2 m^{2}-\ell(\ell+1)}{\sin ^{2} \theta} \mp 2 m(\ell-1) \frac{\cot \theta}{\sin \theta}+\ell(\ell-1) \cot ^{2} \theta, \\
& \beta_{(\ell m)}^{ \pm}(\theta)=2\left[\frac{2 \ell+1}{2 \ell-1}\left(\ell^{2}-m^{2}\right)\right]^{1 / 2}\left( \pm \frac{m}{\sin ^{2} \theta}+\frac{\cot \theta}{\sin \theta}\right) .
\end{aligned}
$$

Finally, in the far field $(r \gg M)$ we decompose the dimensionless Weyl scalar $M r \Psi_{4}$ as

$$
M r \Psi_{4}(t, \vec{r})=\sum_{\ell m}{ }_{-2} C_{\ell m}(t)_{-2} Y_{\ell m}(\theta, \varphi)
$$


where $M$ is the total mass of the binary system (see below for explanations), and $r$ is the radial distance to the binary center of mass. In Eq. (10), and throughout this paper, the notation $\sum_{\ell m}$ is shorthand for $\sum_{\ell=2}^{\infty} \sum_{m=-\ell}^{\ell}$.

\section{B. Details of numerical simulations}

We set up the simulations by placing the $\mathrm{BHs}$ on an initial Cauchy surface using the Brandt-Brügmann prescription [58]; the Hamiltonian constraint is solved using the second-order-accurate multigrid solver AMRMG 59]. We use the Bowen-York [60] framework to incorporate the $\mathrm{BH}$ spins and momenta, with the choice of initial tangential momentum informed by the quasi-circular PN approximation of Ref. [41], Eq.(5.3). These initial conditions typically result in a small level of orbital eccentricity, which is quickly damped by the radiation reaction losses. The simulations described in Ref. 12] showed that the final recoil varied by only a few percent over a wider range of initial eccentricities.

The parameters for the runs considered in this paper are shown in Table \. We use the following notation: EQ and NE indicate equal-mass and unequal-mass runs, respectively. The subscripts $0,+,-$ refer to zero spin, spin aligned, and spin anti-aligned with the orbital angular momentum, respectively (the $\mathrm{EQ}_{\text {planar }}$ run has spins in the orbital plane and anti-aligned with each other). For the unequal-mass cases we use a superscript to indicate the mass ratio $m_{1}: m_{2}$. We denote by $m_{1}$ the $\mathrm{BH}$ horizon mass computed as

$$
m_{1}=\sqrt{m_{\mathrm{irr}, 1}^{2}+\frac{S_{1}^{2}}{4 m_{\mathrm{irr}, 1}^{2}}},
$$

where $\mathbf{S}_{1}=a_{1} m_{1} \hat{\mathbf{S}}_{1}=S_{1} \hat{\mathbf{S}}_{1}$ is the spin angular momentum of $\mathrm{BH} 1, m_{\mathrm{irr}, 1}=\sqrt{A_{1} / 16 \pi}$ is its irreducible mass [61], and $A_{1}$ is its apparent horizon area. Similar definitions hold for BH 2. The binary's total mass is $M=m_{1}+m_{2}, \delta m=m_{1}-m_{2}$, the mass ratio is $q=m_{1} / m_{2} \leq 1$, and the symmetric mass ratio is $\eta=m_{1} m_{2} / M^{2}$. Following Kidder [44], we further define the spin vectors $\mathbf{S}=\mathbf{S}_{1}+\mathbf{S}_{2}, \boldsymbol{\Delta}=M\left(\mathbf{S}_{2} / m_{2}-\mathbf{S}_{1} / m_{1}\right)$, and $\xi=\mathbf{S}+(\delta m / M) \boldsymbol{\Delta}$. The spin vector $\Sigma_{33}^{z}$ is defined below in Sec. VIA.

The mass and spin parameters of the final $\mathrm{BH}$ are $M_{\mathrm{f}}$ and $a_{\mathrm{f}}$. The values of $M_{\mathrm{f}}$ and $a_{\mathrm{f}}$ listed in Table円are computed from the loss of energy and angular momentum from the initial time to the end of the RD phase. They are compatible with the values obtained by extracting the fundamental QNMs (see below Sec. IVB). All spins are orthogonal to the orbital plane, so $\Delta^{x}=\Delta^{y}=0$ (the exception is a single run $\mathrm{EQ}_{\text {planar }}$ with planar spins discussed in Sec. VID In Table [, the spin components in the orbital plane are represented by $\Delta^{p} \equiv\left|\Delta^{x}+i \Delta^{y}\right|$.).
Additionally, all runs have $\left|a_{1}\right| / m_{1}=\left|a_{2}\right| / m_{2}$ with spins pointing in opposite directions, so $\xi \approx 0$ within the accuracy of the initial data.

The simulations were carried out using the moving puncture method [2, 3] in the finite-differencing code Hahndol [62], which solves the Einstein equations in a standard $3+1$ BSSN conformal formulation. Dissipation [63] terms (tapered to zero near the punctures) and constraint-damping [64] terms were added for robust stability. We used the gauge condition recommended in Ref. [65] for moving punctures, fourth-order-accurate mesh-adapted differencing [66] for the spatial derivatives, and a fourth-order-accurate Runge-Kutta algorithm for the time-integration. The adaptive mesh refinement and most of the parallelization was handled by the software package Paramesh [67], with fifth-order accurate interpolation between mesh refinement regions.

The grid spacing in the finest refinement region around each $\mathrm{BH}$ is $h_{f}=3 M / 160$. We extract data for the radiation at a radius $r_{\text {ext }}=45 \mathrm{M}$. The wave extraction was performed by 4 th order interpolation to a sphere followed by angular integration with a Newton-Cotes formula. We have found satisfactory convergence of the results. For example, for the 1:2 mass ratio run, for which a higher resolution of $h_{f}=1 M / 64$ was run in addition to $h_{f}=3 M / 160$, the rates of convergence of the Hamiltonian and momentum constraints are comparable to those found in our equal mass runs reported in [68], and the radiated momenta from the two resolutions agree to within $2 \%$. This was also true for a 2:3 mass ratio test case with aligned spins (the $\mathrm{NE}++$ run in Ref. [12], which is representative of the $\mathrm{NE}_{+-}^{2: 3}$ and $\mathrm{NE}_{-+}^{2: 3}$ runs here).

\section{MULTIPOLAR FORMALISM}

In this Section we review the most relevant results from Thorne [50], showing how a multipole decomposition of the gravitational radiation field can be used to calculate the energy, angular momentum, and linear momentum fluxes from a BH binary system. When restricting the analysis to leading order terms we shall often express the radiative multipole moments in terms of the source multipole moments [51, 52, 53, 54], so in much of the discussion below we will use these two descriptions interchangebly.

\section{A. Linear momentum flux}

In the literature 7, 8, 9, 10, 11, 16] it is common to compute the linear momentum flux, and then the recoil, using the following formula

$$
\frac{d P_{i}}{d t}=\frac{r^{2}}{16 \pi} \int d \Omega \frac{x_{i}}{r}\left|\int_{-\infty}^{t} d t \Psi_{4}\right|^{2}
$$

where $r$ is the extraction radius and the antiderivative of $\Psi_{4}$ is used because the linear momentum flux scales 
TABLE I: Parameters of the numerical simulations (see Sec. IB for explanations). All masses are normalized to an inital total mass of $M=1$.

\begin{tabular}{c|ccccccccccccc}
\hline \hline Run & $m_{1}$ & $m_{2}$ & $\delta m$ & $q$ & $a_{1} / m_{1}$ & $a_{2} / m_{2}$ & $\Delta^{z}$ & $\Delta^{p}$ & $\xi^{z}$ & $\Sigma_{33}^{z}$ & $M_{\mathrm{f}}$ & $a_{\mathrm{f}} / M_{\mathrm{f}}$ & $v_{\mathrm{f}}(\mathrm{km} / \mathrm{s})$ \\
\hline $\mathrm{EQ}_{+-}$ & 0.503 & 0.503 & 0.0 & 1.0 & 0.198 & -0.198 & -0.2 & 0.0 & 0.0 & 0.075 & 0.967 & 0.697 & 90 \\
$\mathrm{EQ}_{\text {planar }}$ & 0.503 & 0.503 & 0.0 & 1.0 & 0.198 & -0.198 & 0.0 & 0.2 & 0.0 & 0.0 & 0.967 & 0.697 & 690 \\
$\mathrm{NE}_{00}^{2: 3}$ & 0.401 & 0.593 & -0.192 & 0.677 & 0.0 & 0.0 & 0.0 & 0.0 & 0.0 & 0.0 & 0.960 & 0.675 & 100 \\
$\mathrm{NE}_{00}^{1: 2}$ & 0.333 & 0.667 & -0.333 & 0.500 & 0.0 & 0.0 & 0.0 & 0.0 & 0.0 & 0.0 & 0.966 & 0.633 & 140 \\
$\mathrm{NE}_{00}^{1: 4}$ & 0.2 & 0.8 & -0.6 & 0.250 & 0.0 & 0.0 & 0.0 & 0.0 & 0.0 & 0.0 & 0.980 & 0.478 & 150 \\
$\mathrm{NE}_{+-}^{2: 3}$ & 0.399 & 0.610 & -0.210 & 0.655 & 0.201 & -0.194 & -0.2 & 0.0 & 0.002 & 0.072 & 0.971 & 0.640 & 190 \\
$\mathrm{NE}_{-+}^{2: 3}$ & 0.399 & 0.610 & -0.212 & 0.653 & -0.201 & 0.193 & 0.2 & 0.0 & -0.002 & -0.072 & 0.967 & 0.704 & 70 \\
\hline \hline
\end{tabular}

as the square of the first derivative of the wave strain, whereas $\Psi_{4}$ is proportional to the second derivative of the strain [see Eq. (7) above]. To study how the different multipole moments contribute to the recoil, we could plug Eq. (10) into Eq. (12), as done, e.g., in Ref. [10]. Here, we prefer to use the expression of the linear momentum flux given in terms of the symmetric and trace-free (STF) radiative mass and current multipole moments, as done in Refs. 50, 51, 52, 53, 54.

Starting from Eq. (4.20') in Ref. [50], we write the linear momentum flux as

$$
\begin{aligned}
F_{j} \equiv \frac{d P_{j}}{d t}=\frac{G}{c^{7}} \sum_{\ell=2}^{\infty}[ & \frac{2(\ell+2)(\ell+3)}{\ell(\ell+1) !(2 \ell+3) ! !}{ }^{(\ell+2)} \mathbf{I}_{j A_{\ell}}{ }^{(\ell+1)} \mathbf{I}_{A_{\ell}}\left(\frac{1}{c}\right)^{2(\ell-2)}+\frac{8(\ell+3)}{(\ell+1) !(2 \ell+3) ! !}{ }^{(\ell+2)} \mathbf{S}_{j A_{\ell}}{ }^{(\ell+1)} \mathbf{S}_{A_{\ell}}\left(\frac{1}{c}\right)^{2(\ell-1)} \\
& \left.+\frac{8(\ell+2)}{(\ell-1)(\ell+1) !(2 \ell+1) ! !} \epsilon_{j p q}{ }^{(\ell+1)} \mathbf{I}_{p A_{\ell-1}}{ }^{(\ell+1)} \mathbf{S}_{q A_{\ell-1}}\left(\frac{1}{c}\right)^{2(\ell-2)}\right]
\end{aligned}
$$

where $\mathbf{I}_{A_{\ell}}\left(\mathbf{S}_{A_{\ell}}\right)$ are the $\ell$-dimensional STF mass (current) tensors and left-hand superscripts represent time derivatives. From these tensors, we can construct the radiative multipole moments $\mathcal{I}^{\ell m}$ and $\mathcal{S}^{\ell m}$ according to the normalization given by Eq. (4.7) of Ref. [50]:

$$
\begin{aligned}
\mathcal{I}^{\ell m}= & \frac{16 \pi}{(2 \ell+1) ! !} \\
& \cdot\left(\frac{(\ell+1)(\ell+2)}{2(\ell-1) \ell}\right)^{1 / 2} \mathbf{I}_{A_{\ell}} \mathbf{Y}_{A_{\ell}}^{\ell m *} \\
\mathcal{S}^{\ell m}= & \frac{-32 \pi \ell}{(\ell+1)(2 \ell+1) ! !} \\
& \cdot\left(\frac{(\ell+1)(\ell+2)}{2(\ell-1) \ell}\right)^{1 / 2} \mathbf{S}_{A_{\ell}} \mathbf{Y}_{A_{\ell}}^{\ell m *},
\end{aligned}
$$

where $\mathbf{Y}_{A_{\ell}}^{\ell m}$ are $\ell$-dimensional STF tensors that are closely related to the usual scalar spherical harmonics by

$$
Y_{\ell m}(\theta, \varphi)=\mathbf{Y}_{i_{1} \cdots i_{\ell}}^{\ell m} n^{i_{1}} \cdots n^{i_{\ell}}
$$

with $n^{i}=(\sin \theta \cos \varphi, \sin \theta \sin \varphi, \cos \theta)^{i}$. Note that the radiative moments $\mathcal{I}^{\ell m}$ and $\mathcal{S}^{\ell m}$ are scalar quantities and have no explicit spatial dependence. To simplify the notation below, we incorporate the $(\ell+1)$ time derivatives into the radiative multipole moments, and define

$$
I^{\ell m} \equiv{ }^{(\ell+1)} \mathcal{I}^{\ell m}, \quad S^{\ell m} \equiv{ }^{(\ell+1)} \mathcal{S}^{\ell m}
$$

By combining Eqs. (13), (14), and (16), we find that at leading order (in a $1 / c$ expansion) the linear momentum flux is given by

$$
\begin{gathered}
F_{x}^{(0)}+i F_{y}^{(0)}=\frac{1}{336 \pi}\left[-14 i S^{21} I^{22 *}+\sqrt{14} I^{31} I^{22 *}-\sqrt{210} I^{22} I^{33 *}+7 i \sqrt{6} I^{20} S^{21 *}-7 i \sqrt{6} S^{20} I^{21 *}+\right. \\
\left.14 i I^{21} S^{22 *}+\sqrt{42} I^{30} I^{21 *}-2 \sqrt{21} I^{20} I^{31 *}-2 \sqrt{35} I^{21} I^{32 *}\right]
\end{gathered}
$$


and

$$
F_{z}^{(0)}=\frac{1}{336 \pi}\left[4 \sqrt{14} \Re\left(I^{31} I^{21 *}\right)-14 \Im\left(I^{21} S^{21 *}\right)+2 \sqrt{35} \Re\left(I^{22} I^{32 *}\right)-28 \Im\left(I^{22} S^{22 *}\right)+3 \sqrt{7} I^{20} I^{30}\right] .
$$

Note that Eq. (17) coincides with Eq. (9) in Ref. [47] when we equate the radiative multipole moments with the source moments [51, 52, 53, 54] and reduce to a circular, non-spinning orbit in the $x-y$ plane. In this case only the first three terms in Eq. (17) survive.

The next highest order terms $\left(1 / c^{2}\right.$ with respect to the leading terms) are proportional to the mass octupoles $I^{3 m}$, or current quadrupoles $S^{2 m}$ :

$$
\begin{aligned}
F_{x}^{(1)}+i F_{y}^{(1)}=\frac{1}{672 \pi} & {\left[-7 i \sqrt{6} S^{32} I^{33 *}-14 \sqrt{6} I^{33} I^{44 *}-4 \sqrt{21} S^{20} S^{31 *}-4 \sqrt{35} S^{21} S^{32 *}-2 \sqrt{210} S^{22} S^{33 *}+\right.} \\
& 2 \sqrt{42} S^{30} S^{21 *}+14 i \sqrt{3} I^{30} S^{31 *}-14 i \sqrt{3} S^{30} I^{31 *}+7 i \sqrt{10} I^{31} S^{32 *}-7 i \sqrt{10} S^{31} I^{32 *}- \\
& 2 \sqrt{105} I^{30} I^{41 *}+6 \sqrt{7} I^{40} I^{31 *}-3 \sqrt{70} I^{31} I^{42 *}+3 \sqrt{14} I^{41} I^{32 *}-21 \sqrt{2} I^{32} I^{43 *}+ \\
& \left.2 \sqrt{14} S^{31} S^{22 *}+\sqrt{42} I^{42} I^{33 *}+7 i \sqrt{6} I^{32} S^{33 *}\right],
\end{aligned}
$$

and

$$
\begin{aligned}
F_{z}^{(1)}=\frac{1}{336 \pi} & {\left[3 \sqrt{7} S^{20} S^{30}+4 \sqrt{14} \Re\left(S^{21} S^{31 *}\right)+2 \sqrt{35} \Re\left(S^{22} S^{32 *}\right)-7 \Im\left(I^{31} S^{31 *}\right)-14 \Im\left(I^{32} S^{32 *}\right)-21 \Im\left(I^{33} S^{33 *}\right)+\right.} \\
& \left.2 \sqrt{21} I^{30} I^{40}+3 \sqrt{35} \Re\left(I^{31} I^{41 *}\right)+6 \sqrt{7} \Re\left(I^{32} I^{42 *}\right)+7 \sqrt{3} \Re\left(I^{33} I^{43 *}\right)\right]
\end{aligned}
$$

Note that all of the terms in Eqs. (17) and (19) contain products of multipoles with $m^{\prime}=m \pm 1$, while the terms in Eqs. (18) and (20) have $m^{\prime}=m$, as with familiar quantum-mechanical operators that involve similar $x_{i^{-}}$ weighted integrations over the sphere. Also note that for mass-mass and current-current terms, $\ell^{\prime}=\ell \pm 1$, while for mass-current terms, $\ell^{\prime}=\ell$.

The above formulae (17)-20) are valid for completely general orbits, including eccentricity, spin terms and even for binary systems precessing out of the plane. However, we can simplify them significantly by rotating into the frame where the instantaneous orbital angular momentum is along the $z$-axis. Furthermore, by assuming that terms proportional to $\ddot{R}$ ( $R$ being the binary radial separation) are negligible, we find $I^{20}=I^{30}=S^{30}=I^{32}=$ $I^{40}=I^{41}=I^{43}=0$. In the approximation of $\ddot{R}=0$, the inclusion of terms linear in $\dot{R} \neq 0$ adds no new multipole modes. In fact, one of the primary reasons the derivations above begin with the mass and current tensors $\mathbf{A}_{A_{\ell}}$ and $\mathbf{S}_{A_{\ell}}$ is to facilitate the calculation of the individual radiative moments $I^{\ell m}$ and $S^{\ell m}$ and also identify the contributions from $\dot{R}$ and $\ddot{R}$ terms from a generalized binary orbit 44]. In the case of non-spinning BHs, the formulae (17)-20) can be additionally simplified by setting $S^{20}=I^{21}=S^{22}=S^{31}=S^{33}=0$. Quite interestingly, we obtain that the latter conditions are also valid in the special case of non-precessing BHs where the spins are aligned or anti-aligned with the orbital angular momentum. Since these are the cases we consider in this paper, we refer often to the following approximate formula for the linear momentum flux:

$$
F_{x}+i F_{y} \simeq \frac{1}{672 \pi}\left[-28 i S^{21} I^{22 *}-2 \sqrt{210} I^{22} I^{33 *}-14 \sqrt{6} I^{33} I^{44 *}+2 \sqrt{14} I^{31} I^{22 *}-7 i \sqrt{6} S^{32} I^{33 *}\right], \quad F_{z}=0 .
$$

As we will see below in Sec. IVA, the linear momentum flux contributions from $I^{31} I^{22 *}$ as well as other higher- $\ell$ modes are typically smaller by at least an order of magnitude. When integrating Eq. (21) to get the recoil ve- locity, we also find that (due in large part to the relative phases between the modes) the contribution from $S^{32} I^{33 *}$ is rather minimal. Thus for most of the analysis that follows, we will focus solely on the first three terms 
of Eq. (21).

In the following, sometimes we will use

$$
\mathbf{F}=\left\{F_{x}, F_{y}, F_{z}\right\}, \quad \hat{\mathbf{F}}=\frac{\mathbf{F}}{|\mathbf{F}|} .
$$

All the non-precessing numerical simulations we will analyze have $F_{z}=0$, so we can introduce a complex scalar flux

$$
F \equiv F_{x}+i F_{y} .
$$

Since what we extract from the numerical simulations are the modes ${ }_{2} C_{\ell m}$ computed over the sphere surrounding the binary, we need to relate the ${ }_{-2} C_{\ell m}$ to the radiative mass and current multipole moments defined above. From Eq.(4.3) of [50],

$$
h=\sum_{\ell m}\left({ }^{(\ell)} \mathcal{I}^{\ell m} T_{a b}^{E 2, \ell m} m^{a} m^{b}+{ }^{(\ell)} \mathcal{S}^{\ell m} T_{a b}^{B 2, \ell m} m^{a} m^{b}\right),
$$

where $h \equiv h_{a b} m^{a} m^{b}$ and $h_{a b}$ is the metric perturbation $g_{a b}-\eta_{a b}$ in the transverse traceless gauge, which satisfies Eq. (5), and $T_{a b}^{E 2, \ell m}$ and $T_{a b}^{B 2, \ell m}$ are the "pure-spin" harmonics of Thorne. From Appendix A of [69],

$$
\begin{aligned}
T_{a b}^{E 2, \ell m} & =\frac{1}{\sqrt{2}}\left({ }_{-2} Y^{\ell m} m_{a} m_{b}+{ }_{2} Y^{\ell m} m_{a}^{*} m_{b}^{*}\right) \\
T_{a b}^{B 2, \ell m} & =\frac{-i}{\sqrt{2}}\left({ }_{-2} Y^{\ell m} m_{a} m_{b}-{ }_{2} Y^{\ell m} m_{a}^{*} m_{b}^{*}\right) .
\end{aligned}
$$

Substituting Eqs. (25a)-(25b) into Eq. (24) and recalling that $m^{a} m_{a}=0$ gives

$$
h=\frac{1}{\sqrt{2} r} \sum_{\ell m}\left({ }^{(\ell)} \mathcal{I}^{\ell m}+i^{(\ell)} \mathcal{S}^{\ell m}\right)+2 Y^{\ell m}
$$

Now taking the complex conjugate and using the fact that ${ }_{+2} Y^{* \ell m}=(-1)^{m}{ }_{-2} Y^{\ell-m}$ [note there is a typo in Eq. (3.1) of Ref. [56]] we obtain

$$
\begin{aligned}
h^{*} & =\frac{1}{\sqrt{2} r} \sum_{\ell m}(-1)^{m}\left({ }^{(\ell)} \mathcal{I}^{\ell m *}-i^{(\ell)} \mathcal{S}^{\ell m *}\right)_{-2} Y^{\ell-m} \\
& =\frac{1}{\sqrt{2} r} \sum_{\ell m}(-1)^{m}\left({ }^{(\ell)} \mathcal{I}^{\ell-m *}-i^{(\ell)} \mathcal{S}^{\ell-m *}\right)_{-2} Y^{\ell m} .
\end{aligned}
$$

Using the tetrad choice of Eqs. (1a)-17), $\partial^{2} h^{*} / \partial t^{2}=\ddot{h}_{+}-$ $i \ddot{h}_{\times}=-\Psi_{4}$, which decomposed into spin -2 weighted harmonics, gives

$$
\frac{\partial^{2} h^{*}}{\partial t^{2}}=-\frac{1}{M r} \sum_{\ell m}{ }_{-2} C_{\ell m-2} Y^{\ell m},
$$

allowing us to see term-by-term that

$$
(-1)^{m}\left({ }^{(\ell+2)} \mathcal{I}^{\ell-m *}-i^{(\ell+2)} \mathcal{S}^{\ell-m *}\right)=-\sqrt{2}_{-2} C_{\ell m} .
$$

Recall that $(-1)^{m} \mathcal{I}^{\ell-m *}=\mathcal{I}^{\ell m}$ and $(-1)^{m} \mathcal{S}^{\ell-m *}=$ $\mathcal{S}^{\ell m}$, which allows us to write

$$
\begin{aligned}
{ }^{(\ell+2)} \mathcal{I}^{\ell m} & =-\frac{1}{\sqrt{2}}\left[{ }_{-2} C_{\ell m}+(-1)^{m}{ }_{-2} C_{\ell-m}^{*}\right] \\
{ }^{(\ell+2)} \mathcal{S}^{\ell m} & =-\frac{i}{\sqrt{2}}\left[{ }_{-2} C_{\ell m}-(-1)^{m}{ }_{-2} C_{\ell-m}^{*}\right]
\end{aligned}
$$

Equations (17)-21) are expressed in terms of $I^{\ell m} \equiv$ ${ }^{(\ell+1)} \mathcal{I}^{\ell m}$ and $S^{\ell m} \equiv{ }^{(\ell+1)} \mathcal{S}^{\ell m}$, which can be computed by integrating Eqs. (30a), (30b) once in time. To avoid the complication of an undetermined constant of integration, we typically integrate ${ }_{-2} C_{\ell m}(t)$ backwards in time, since in the numerical data (and what we expect happens in reality) all the moments go to zero exponentially after the merger. At early times on the other hand, most of the modes are significantly non-zero and also include a large amount of numerical noise due to the initial conditions.

\section{B. Energy and angular momentum flux}

Unlike the equations for the linear momentum flux, which all involve "beating" between pairs of different modes, the energy and angular-momentum flux expressions involve terms of the form $\left|I^{\ell m}\right|^{2}$, allowing us to isolate the individual contributions from each mode. As we will see below, for the comparable-mass binary systems that we analyse $\left(m_{1}: m_{2}=1: 1,2: 3,1: 2\right)$, the amplitude of the mass quadrupole moment $I^{22}$ is roughly an order of magnitude larger than the next largest mode. Thus it almost completely dominates the energy and angular momentum fluxes, and we can write [see Eq. (4.16) in Ref. [50]]

$$
\frac{d E}{d t}=\frac{1}{32 \pi} \sum_{\ell m}\left(\left|I^{\ell m}\right|^{2}+\left|S^{\ell m}\right|^{2}\right) \simeq \frac{1}{16 \pi}\left|I^{22}\right|^{2} .
$$

The multipole expressions for angular momentum flux are somewhat more complicated, but for the numerical simulations considered in this paper, the only non-zero modes have $\ell+m$ even for $I^{\ell m}$ and $\ell+m$ odd for $S^{\ell m}$, so we can neglect the $(m, m \pm 1)$ cross-terms in Eq. (4.23) of Ref. 50]. These cross-terms are responsible for angular momentum loss in the $x-y$ plane, so it is reasonable that they must be zero for non-precessing planar orbits. In this case, where the angular momentum is solely along the $\hat{\mathbf{z}}$-axis, we have

$$
\begin{aligned}
\frac{d J_{z}}{d t} & =\frac{i}{32 \pi} \sum_{\ell m} m\left({ }^{(\ell)} \mathcal{I}^{\ell m *(\ell+1)} \mathcal{I}^{\ell m}+{ }^{(\ell)} \mathcal{S}^{\ell m *(\ell+1)} \mathcal{S}^{\ell m}\right) \\
& \simeq-\frac{1}{8 \pi} \Im\left[{ }^{(2)} \mathcal{I}^{22 *(3)} \mathcal{I}^{22}\right],
\end{aligned}
$$

where we have restored the explicit time derivatives as in Eq. (16). 
TABLE II: Energy and angular momentum radiated in each of the dominant multipole modes. In parentheses we show the amount radiated only after the peak of GW energy flux. All units are normalized to $M=1$.

\begin{tabular}{c|cccccccccc}
\hline \hline Run & $\begin{array}{c}E_{22} \\
\left(\times 10^{-2}\right)\end{array}$ & $\begin{array}{c}E_{21} \\
\left(\times 10^{-4}\right)\end{array}$ & $\begin{array}{c}E_{32} \\
\left(\times 10^{-4}\right)\end{array}$ & $\begin{array}{c}E_{33} \\
\left(\times 10^{-4}\right)\end{array}$ & $\begin{array}{c}E_{44} \\
\left(\times 10^{-4}\right)\end{array}$ & $\begin{array}{c}J_{22} \\
\left(\times 10^{-1}\right)\end{array}$ & $\begin{array}{c}J_{21} \\
\left(\times 10^{-4}\right)\end{array}$ & $\begin{array}{c}J_{32} \\
\left(\times 10^{-4}\right)\end{array}$ & $\begin{array}{c}J_{33} \\
\left(\times 10^{-3}\right)\end{array}$ & $\begin{array}{c}J_{44} \\
\left(\times 10^{-3}\right)\end{array}$ \\
\hline $\mathrm{EQ}_{+-}$ & 3.5 & 0.22 & 1.6 & 0.04 & 3.3 & 2.2 & -0.70 & 7.9 & -0.02 & 1.9 \\
& $(1.4)$ & $(0.17)$ & $(1.2)$ & $(0.02)$ & $(1.5)$ & $(0.50)$ & $(-0.46)$ & $(-2.0)$ & $(-0.01)$ & $(0.64)$ \\
$\mathrm{NE}_{00}^{2: 3}$ & 3.1 & 0.61 & 0.90 & 5.6 & 2.9 & 2.2 & -2.1 & 3.9 & -3.1 & 1.8 \\
& $(1.1)$ & $(0.40)$ & $(0.66)$ & $(2.8)$ & $(1.0)$ & $(0.45)$ & $(-0.98)$ & $(2.5)$ & $(-1.1)$ & $(0.46)$ \\
$\mathrm{NE}_{00}^{1: 2}$ & 2.5 & 1.4 & 0.47 & 12.0 & 2.7 & 1.8 & -4.8 & 2.4 & -6.9 & 1.7 \\
& $(0.87)$ & $(0.94)$ & $(0.30)$ & $(5.8)$ & $(0.73)$ & $(0.37)$ & $(-2.4)$ & $(1.3)$ & $(-2.3)$ & $(0.30)$ \\
$\mathrm{NE}_{00}^{1: 4}$ & 1.2 & 2.1 & 0.27 & 16.0 & 3.3 & 1.2 & -8.0 & 1.6 & -11.0 & 2.4 \\
& $(0.35)$ & $(1.4)$ & $(0.09)$ & $(6.6)$ & $(1.2)$ & $(0.16)$ & $(-3.8)$ & $(0.27)$ & $(-2.9)$ & $(0.48)$ \\
$\mathrm{NE}_{+-}^{2: 3}$ & 2.9 & 1.6 & 0.93 & 5.2 & 2.6 & 2.0 & -5.4 & 2.1 & -2.9 & 1.6 \\
& $(1.0)$ & $(1.0)$ & $(0.67)$ & $(2.5)$ & $(0.82)$ & $(0.31)$ & $(-2.9)$ & $(5.3)$ & $(-0.98)$ & $(0.33)$ \\
$\mathrm{NE}_{-+}^{2: 3}$ & 3.3 & 0.14 & 1.1 & 7.1 & 2.9 & 2.3 & -0.50 & 4.4 & -3.9 & 1.8 \\
& $(1.1)$ & $(0.09)$ & $(0.78)$ & $(3.4)$ & $(0.92)$ & $(0.44)$ & $(-0.21)$ & $(3.1)$ & $(-1.3)$ & $(0.37)$ \\
\hline \hline
\end{tabular}

Integrating Eqs. (31) and (32) term-by-term, we can calculate how much energy and angular momentum are radiated in each of the dominant modes, similar to the approach of Ref. [75]. We introduce the quantities $E_{\ell m}$ and $J_{\ell m}$ as the total energy and angular momentum radiated in each $(\ell, m)$ mode, computed by integrating Eqs. (31) and (32) in time, term by term (for conciseness, we combine both the $m$ and $-m$ terms into $E_{\ell m}$ and $J_{\ell m}$ and restrict our notation to $m>0$ ). Note that while $E_{\ell m}$ is always positive, $J_{\ell m}$ can also be negative, corresponding to angular momentum in the $-\hat{z}$ direction. These results are shown in Table II along with the contributions from just the RD phase $\left(t>t_{\text {peak }}\right.$, where $t_{\text {peak }}$ is the point at which $\left|I^{22}\right|$ reaches its peak, closely corresponding to the peak in GW energy emission). We will see below in Section $\mathrm{V}$ that these various energy contributions agree closely with the Newtonian predictions for the relative mass-scalings. For example, the energy $E_{22}$ in the inspiral phase should scale as $\eta$, while the RD contribution should scale like $\eta^{2}$. It is important to note that the different moments have different scalings: $E_{33} \sim \eta^{2} \delta m^{2}$, while the $I^{44}$ contribution has a much weaker dependence on mass ratio: $E_{44} \sim \eta^{2}(1-3 \eta)^{2}$.

In the limit of very large initial separation (small initial frequency), each of the $E_{\ell m}$ and $J_{\ell m}$ should converge to a finite value, with the notable exception of $J_{22}$. It is well-know that the angular momentum of a binary system scales as $R^{1 / 2}$, and is thus unbound in the limit of $R \rightarrow \infty$, but it is interesting to see that the higher-order contributions to the angular momentum all converge at large $R$. This can be understood directly from Eq. (32) in the Keplerian limit of $R=M^{1 / 3} \omega^{-2 / 3}$. At leading order, radiation reaction follows the relation $d t \sim \omega^{-11 / 3} d \omega$ so the angular momentum in the inspiral is

$$
\begin{aligned}
J_{22} & =\frac{1}{8 \pi} \int_{t=-\infty}^{t_{0}} d t \Im\left[{ }^{(2)} \mathcal{I}^{22 *(3)} \mathcal{I}^{22}\right] \\
& \sim \int_{\omega=0}^{\omega_{0}} \omega^{2 / 3} \omega^{5 / 3} \omega^{-11 / 3} d \omega \rightarrow \infty
\end{aligned}
$$

As we will see below in Section V] for all the other energy and angular momentum modes, the fluxes from Eqs. (31), 32 scale as $\omega^{10 / 3}$ or higher powers, and thus converge when integrated over $\omega^{-11 / 3} d \omega$.

\section{MULTIPOLE ANALYSIS OF THE NUMERICAL SIMULATIONS}

In this Section we want to investigate how the different multipole moments evolve during the inspiral and ringdown phases of $\mathrm{BH}$ binary mergers.

\section{A. Inspiral phase}

As can be derived in PN theory 36 and has been confirmed numerically in Refs. [2, [3], the $\ell=2, m=2$ mode in Eq. (10) is circularly polarized to leading order throughout the coalescence. Because of this, Ref. 73. defined the (dominant) orbital angular frequency as

$$
\omega_{\mathrm{D}}^{\ell m}=-\frac{1}{m} \Im\left(\frac{{ }_{2} \dot{C}_{\ell m}}{{ }_{-2} C_{\ell m}}\right) .
$$

Here, we extend Eq. (34) by defining several (dominant) orbital angular frequencies, each of them being related to 
a specific multipole moment, $I^{\ell m}$ or $S^{\ell m}$, as

$$
\omega_{\mathrm{D}}^{I \ell m}=-\frac{1}{m} \Im\left(\frac{\dot{I}^{\ell m}}{I^{\ell m}}\right), \quad \omega_{\mathrm{D}}^{S \ell m}=-\frac{1}{m} \Im\left(\frac{\dot{S}^{\ell m}}{S^{\ell m}}\right) .
$$

We plot these frequencies in Fig. 2 for the dominant multipole moments $I^{22}, S^{21}, I^{33}, I^{44}$, and $S^{32}$, for the $\mathrm{NE}_{00}^{2: 3}$ (left panel) and $\mathrm{NE}_{00}^{1: 2}$ (right panel) runs. The amplitudes of the $I^{31}$ and $I^{42}$ modes are too weak and dominated by noise to extract a dominant frequency. In this figure, as well as most shown in the rest of the paper, we plot the time variable with respect to $t_{\text {peak }}$. We notice that the frequencies corresponding to the modes with $\ell=m$ agree quite well throughout the inspiral and ringdown, but the frequency of the $S^{21}$ mode decouples from the others approximately $50 M$ before the peak in the $I^{22}$ mode. As we shall see in Sec. VI this is due to the fact that, during the ringdown phase, the dominant angular frequency associated to the $S^{21}$ mode is almost twice as large as those of the other leading modes 70, 71, 72]. This decoupling plays a major role in determining the shape of the kick and anti-kick (see Sec. VI below), and also suggests that the transition to RD may begin long before the peak of the GW flux. Similarly, the $S^{32}$ mode should converge to a higher RD frequency $\left(\omega_{320} / 2 \simeq 0.37 / M_{\mathrm{f}}\right.$ for these runs), but may be limited by numerical noise here, as well as possible mode mixing with the dominant $I^{22}$ moment.

In Fig. 3 we show the amplitudes of the multipole moments in Eq. (21). Again, the left panel refers to the $\mathrm{NE}_{00}^{2: 3}$ run, while the right panel to the $\mathrm{NE}_{00}^{1: 2}$ run. The mass-quadrupole moment $I^{22}$ clearly dominates in both cases, while the $I^{31}$ and $I^{42}$ modes are so weak as to be almost completely overwhelmed by numerical noise. In addition to having dissimilar amplitudes, the different moments also peak at slightly different times, which may be related to the fact that $\mathrm{RD}$ modes are excited at different times. In particular, the modes mentioned above with $\ell \neq m$ tend to peak later in time, perhaps due to a longer transition to the higher QNM frequency. As we shall see in Sec. V] as the mass ratio becomes more extreme (i.e., decreasing $\eta$ ), the higher-order modes increase in relative amplitude, with $I^{33}$ and $S^{21}$ both proportional to $\eta \delta m$. $I^{44}$ and $S^{32}$, however, scale as $\eta(1-3 \eta)$, so they increase only slightly in the range of masses considered here.

Next, in Fig. 4, we show the amplitude of the linear momentum flux from the mode-pairs included in Eq. (21). Here we define the complex flux $F^{21,22}=$ $(-14 i / 336 \pi) S^{21} I^{22 *}$ and other $F^{\ell m, \ell^{\prime} m^{\prime}}$ analogously from Eq. (21). As in Fig. 3, the mass-quadrupole terms dominate, with significantly smaller contributions from the $S^{32}$ and $I^{31}$ modes. However, note the appreciable flux amplitude from the $F^{33,44} \sim I^{33} I^{44 *}$ term, which is formally a higher-order correction in a $(1 / c)$ expansion [46, 47]. From Fig. 4 we expect that the first three pairs of modes in Eq. (21) should contribute most significantly to the recoil. Including the complex phase relations between the different modes, we find this result will be supported further by the analysis in Sec. VIA.

\section{B. Ringdown phase}

We now extract the QNMs, notably the fundamental and the first two overtones, present in the most significant multipole moments during the RD phase. We follow the procedure outlined in Ref. 73]. To avoid possible constant offsets introduced by integrating Eqs. (30a), (30b), we prefer to extract the QNMs directly from the ${ }_{-2} C_{\ell m}$ instead of using $I_{\ell m}$ or $S_{\ell m}$. Additionally, from Eqs. (30a), (30b), we see that ${ }^{(1)} I^{\ell m}$ and ${ }^{(1)} S^{\ell m}$ are made up of both ${ }_{-2} C_{\ell m}$ and ${ }_{-2} C_{\ell-m}$, which in general do not have the same QNM frequencies, so it is more reliable to extract the RD modes from just ${ }_{-2} C_{\ell m}$ (however, in practice we find that the RD phase is dominated by modes with positive $m$ ). Following the approach of Ref. [72], we define the complex frequencies $\sigma_{\ell m n}$ :

$$
\sigma_{\ell m n} \equiv \omega_{\ell m n}-i / \tau_{\ell m n}
$$

and each $\mathrm{RD}$ mode is proportional to $\exp \left(-i \sigma_{\ell m n} t\right)$. In this notation, $\omega_{\ell m n}$ are the QNM oscillation frequencies [not to be confused with the dominant frequencies of Eq. [35] ] and $\tau_{\ell m n}$ are the mode decay times, all functions of the final black hole mass and spin. The subscripts $\ell$ and $m$ are the same spherical wavenumbers used above, and $n=0$ denotes the fundamental mode, with $n=1,2, \cdots$, corresponding to the higher overtones. The fundamental QNM frequencies $\sigma_{\ell m 0}$ are listed in Table for the NR runs listed above. All frequencies and decay times are measured in units of the final mass $M_{\mathrm{f}}$.

We present the $\mathrm{RD}$ analysis only for the $\mathrm{NE}_{00}^{2: 3}$ run, but the others are qualitatively very similar. We have extracted the various QNM contributions to the ${ }_{2} C_{\ell m}$ $\mathrm{RD}$ signal in the following way (see also Ref. $73[$ ): We expect that at late times the $n=0 \mathrm{QNM}$ dominates. We fit the signal after time $t_{\text {peak }}+t_{r}$ to this single mode using non-linear regression and choose $t_{r}$ to minimize the error in the fit. We have four dimensionless parameters in this non-linear fit: the QNM amplitude and phase, $\mathcal{C}_{\ell m 0}$ and $\phi_{\ell m 0}$, and the QNM frequency and decay time $M \omega_{\ell m 0}$ and $\tau_{\ell m 0} / M$. However, instead of fitting directly for these four parameters, we treat $M \omega_{\ell m 0}$ and $\tau_{\ell m 0} / M$ as functions of $a_{\mathrm{f}} / M_{\mathrm{f}}$ and $M_{\mathrm{f}} / M$ (which can be obtained via interpolation from tabulated values given in Ref. 72] ). The advantage of using $\left(a_{\mathrm{f}} / M_{\mathrm{f}}, M_{\mathrm{f}} / M, \mathcal{C}_{\ell m 0}, \phi_{\ell m 0}\right)$ for the set of fitting parameters comes when we fit to higher overtones. As done in Ref. [73], we extract the QNMs treating the real and imaginary parts of ${ }_{-2} C_{\ell m}$ as independent. Below we shall list results obtained from $\operatorname{Re}\left[{ }_{-2} C_{\ell m}\right]$.

By applying this procedure to the dominant mode, ${ }_{-2} C_{22}$, we obtain $a_{\mathrm{f}} / M_{\mathrm{f}}=0.669$ and $M / M_{\mathrm{f}}=0.965$ together with the amplitude and phase of the fundamental 

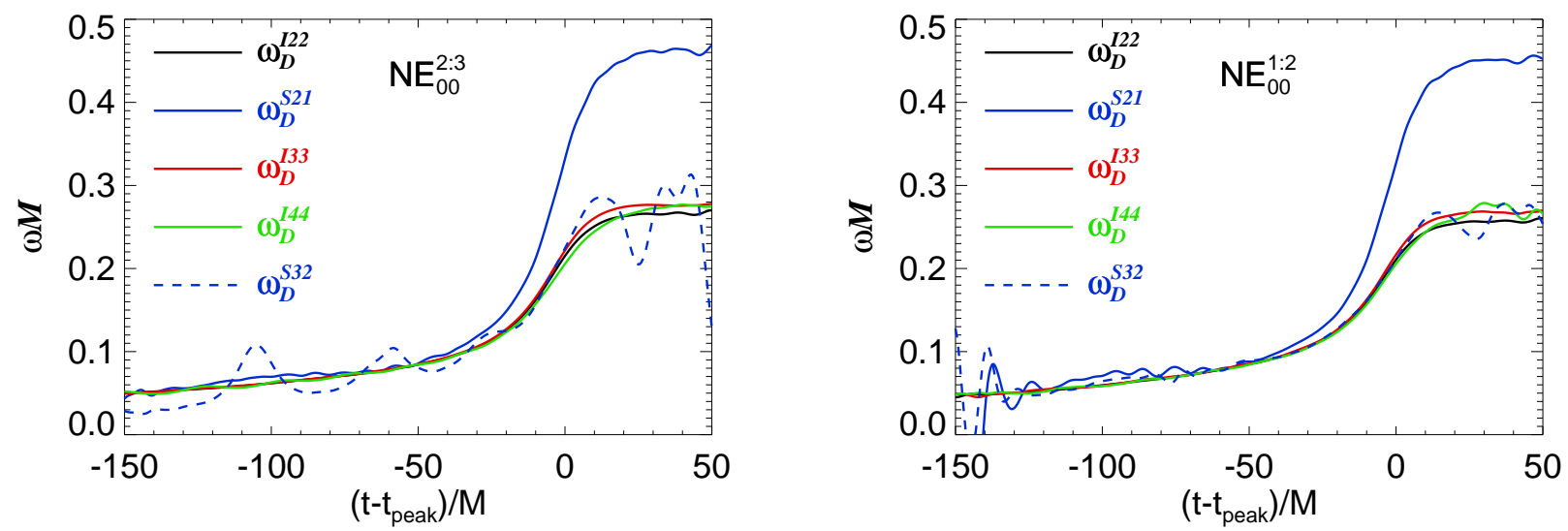

FIG. 2: Dominant orbital angular frequency obtained from the individual radiative multipole moments, as determined by Eq. (35). The different frequencies with $\ell=m$ agree closely throughout the inspiral and RD phases. The frequency with $\ell=2, m=1$ decouples from the others at earlier time and reaches a much higher plateau. The left panel refers to the NE $\mathrm{E}_{00}^{2: 3}$ run and the right panel to the $\mathrm{NE}_{00}^{1: 2}$ run. We denote with $t_{\text {peak }}$ the time at which $I^{22}$ reaches its maximum.
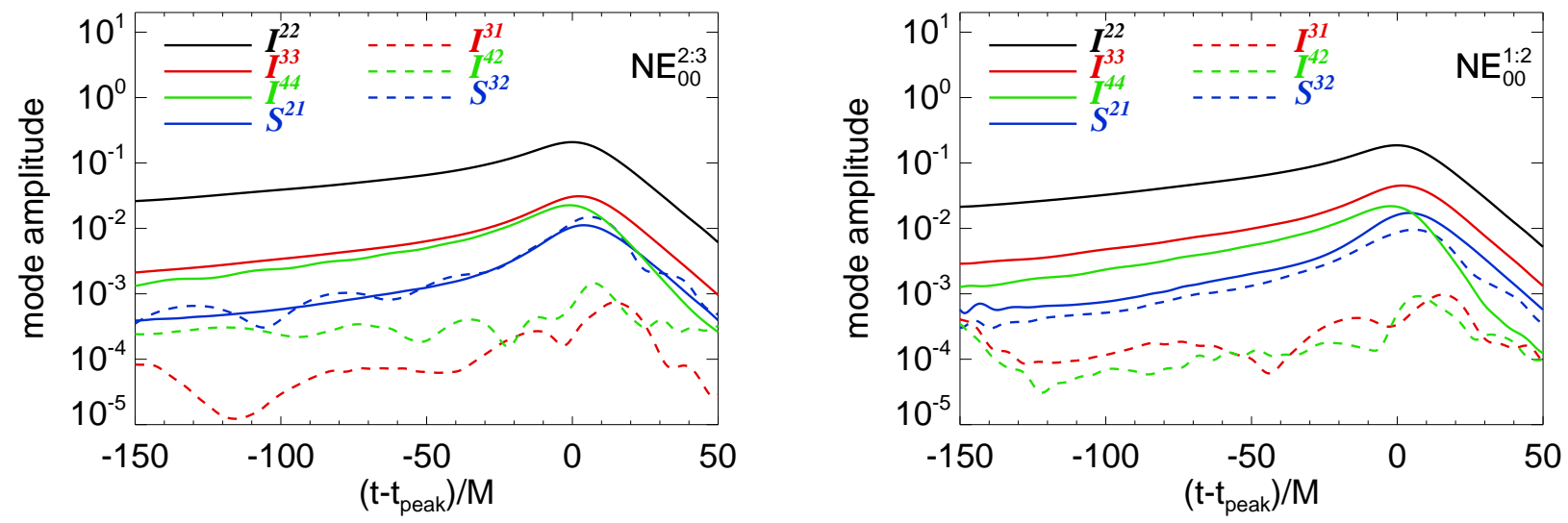

FIG. 3: Amplitudes of the dominant radiative multipole moments. On the left panel we show the modes for the NE $\mathrm{E}_{00}^{2: 3}$ run, while on the right panel the modes for the $\mathrm{NE}_{00}^{1: 2}$ run. The leading-order mass quadrupole $I^{22}$ is about an order of magnitude stronger than any other mode. The oscillating behavior of the $S^{32}$ moment during RD is likely due to mode mixing with $I^{22}$. We denote with $t_{\text {peak }}$ the time at which $I^{22}$ reaches its maximum.

TABLE III: Frequencies and decay times for the fundamental QNMs for each of the numerical simulations. $\omega_{\ell m 0}$ is in units of $M_{\mathrm{f}}^{-1}$ and $\tau_{\ell m 0}$ is in units of $M_{\mathrm{f}}$.

\begin{tabular}{|c|c|c|c|c|c|c|c|c|c|c|}
\hline Run & $\bar{a} / M_{\mathrm{f}}$ & $\omega_{210} \tau_{210}$ & $\omega_{220}$ & $\tau_{220}$ & $\omega_{320}$ & $\tau_{320}$ & $\omega_{330}$ & $\tau_{330}$ & $\omega_{440}$ & $\tau_{440}$ \\
\hline$\overline{\mathrm{EQ}_{+}}$ & 0.697 & $0.454 \quad 12.2$ & 0.531 & 12.4 & 0.758 & 11.9 & 0.841 & 12.0 & 1.14 & $\overline{11.8}$ \\
\hline $\mathrm{NE}_{00}^{2: 3}$ & 0.675 & $\begin{array}{lll}0.450 & 12.1\end{array}$ & 0.521 & 12.2 & 0.749 & 11.7 & 0.827 & 11.9 & 1.12 & 11.7 \\
\hline $\mathrm{NE}_{00}^{1: 2}$ & 0.633 & $0.442 \quad 11.9$ & 0.505 & 12.1 & 0.734 & 11.6 & 0.803 & 11.7 & 1.09 & 11.5 \\
\hline $\mathrm{NE}_{00}^{1: 4}$ & 0.423 & 0.41111 .5 & 0.445 & 11.5 & 0.674 & 11.1 & 0.711 & 11.1 & 0.963 & 10.9 \\
\hline $\mathrm{NE}_{+-}^{2: 3}$ & 0.640 & $0.443 \quad 11.9$ & 0.507 & 12.1 & 0.736 & 11.6 & 0.806 & 11.7 & 1.09 & 11.5 \\
\hline $\mathrm{NE}_{-+}^{2: 3}$ & 0.704 & $0.456 \quad 12.2$ & 0.533 & 12.4 & 0.760 & 11.9 & 0.845 & 12.1 & 1.14 & 11.9 \\
\hline
\end{tabular}



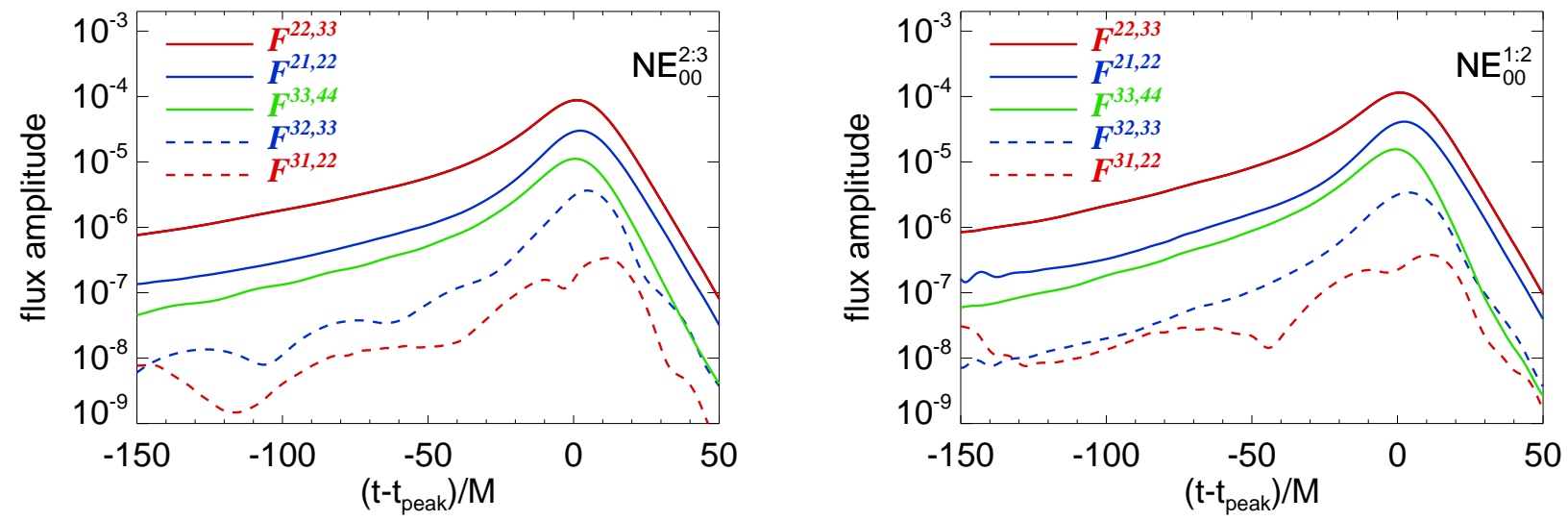

FIG. 4: Linear momentum flux of the strongest radiative multipole moments, i.e., the ones in Eq. (21). On the left panel we show the modes for the $\mathrm{NE}_{00}^{2: 3}$ run, while on the right panel the modes for the $\mathrm{NE}_{00}^{1: 2}$ run. We denote with $t_{\mathrm{peak}}$ the time at which $I^{22}$ reaches its maximum. 
QNM. We include additional overtones $(n>0)$ successively. For each value of $n$, we refit the entire function, so for $n=0$ there are 4 parameters in the fit, for $n=1$ there are 6 , for $n=2$ there are 8 , and so forth. Thus, applying a 6-parameter fit we successfully extract also the first overtone simultaneously, obtaining slightly different values for $a_{\mathrm{f}} / M_{\mathrm{f}}=0.661$ and $M / M_{\mathrm{f}}=0.958$. We find it impossible to extract, with a single 8-parameter fit, also the second overtone. By contrast if we keep $a_{\mathrm{f}} / M_{\mathrm{f}}$ and $M / M_{\mathrm{f}}$ fixed and equal to the values obtained when extracting the fundamental QNM, we find that we can fit up to the second overtone. Moreover, quite interestingly, the fit provides waveforms that compare very well with the NR waveforms up to the peak of $I_{22}$, as can be seen in the upper left panel of Fig. 5 .

The remaining panels in Fig. 5] show results for the other relevant modes ${ }_{-2} C_{33},{ }_{-2} C_{44}$ and ${ }_{-2} C_{32}$. As obtained in Ref. [73], we find a "mode-mixing" in ${ }_{-2} C_{32}$, i.e., the $\mathrm{RD}$ waveform is a combination of $\ell=2, m=2$ and $\ell=3, m=2$ QNMs. This effect appears to be most important between modes with the same $m$ value, and may possibly be explained by the fact that the QNMs should really be expressed as spheroidal, not spherical harmonics [72, 73]. Including both sets of modes means that the ${ }_{-2} C_{32}$ is actually fit using 14 parameters: the final mass and spin, and the amplitude and phase of 6 QNMs.

By fitting the fundamental QNM for each ringdown waveform, we obtain $a_{\mathrm{f}} / M_{\mathrm{f}}=0.671$ and $M / M_{\mathrm{f}}=0.972$; $a_{\mathrm{f}} / M_{\mathrm{f}}=0.527$ and $M / M_{\mathrm{f}}=0.884 ; a_{\mathrm{f}} / M_{\mathrm{f}}=0.686$ and $M / M_{\mathrm{f}}=0.981$, for ${ }_{-2} C_{33},{ }_{-2} C_{44}$ and ${ }_{-2} C_{32}$, respectively. We also are able to extract the fundamental QNM for the ${ }_{-2} C_{21}$ mode (not shown in Fig. 5) and find $a_{\mathrm{f}} / M_{\mathrm{f}}=0.678$ and $M / M_{\mathrm{f}}=0.960$. All of these values for the inferred final $\mathrm{BH}$ spin and mass are rather consistent, except for ${ }_{-2} C_{44}$. This discrepancy might be due to numerical resolution effects, and will be the object of future investigations.

Thus we find that although we cannot simultaneously extract three QNMs (the fundamental and two overtones) and we are not able to clearly determine the onset of the $\mathrm{RD}$ phase, we do obtain that for $t>t_{\text {peak }}$ the numerical waveforms can be well fitted by a superposition of three QNMs. This result explains why the simple matching procedure from inspiral to RD adopted in the EOB model 39, 47, 73] can almost always work succesfully (see Ref. 77] for some caveats). In Sec. $\mathrm{VB}$ we shall adopt the same matching procedure of the EOB model when building the full waveform using the pseudo-analytic model of Sec. V.

\section{EFFECTIVE NEWTONIAN MODEL}

In an attempt to better understand the amplitudes and frequencies of the various modes during the inspiral and merger phases, we present here what we call the "effective Newtonian" (eN) model. It begins with calculating the leading-order Newtonian formulae for each multipole moment of the source, as a function of the BH masses, binary separation $R$, and orbital phase $\phi$. To extend these formulae through the end of the inspiral and into the merger phase, we introduce an effective radial separation to absorb PN effects into the leading-order multipole expressions. Each multipole moment is then individually matched to a linear superposition of ringdown modes, as is done in the effective-one-body model [39, 47, 73]. Taken together with the match to Kerr QNMs, this eN model provides an excellent framework within which we can understand the details of the linear momentum flux and net recoil velocity.

\section{A. Newtonian Multipole Moments}

Working at leading Newtonian order for each mode, we equate the radiative multipole moments to the source multipole moments. Restricting ourselves to circular, planar orbits, we find that for non-spinning systems, the dominant modes are 51, 52, 53, 54]

$$
\begin{aligned}
& S_{\text {nospin }}^{21}=-\frac{8}{3} i \sqrt{\frac{2 \pi}{5}} \frac{\delta m}{M} \mu R^{3} \omega^{4} e^{-i \phi} \\
& I_{\text {nospin }}^{22}=16 i \sqrt{\frac{2 \pi}{5}} \mu R^{2} \omega^{3} e^{-2 i \phi} \\
& I_{\text {nospin }}^{31}=-\frac{2}{3} \sqrt{\frac{\pi}{35}} \frac{\delta m}{M} \mu R^{3} \omega^{4} e^{-i \phi} \\
& S_{\text {nospin }}^{32}=-\frac{16}{3} \sqrt{\frac{2 \pi}{7}} \mu(1-3 \eta) R^{4} \omega^{5} e^{-2 i \phi} \\
& I_{\text {nospin }}^{33}=54 \sqrt{\frac{\pi}{21}} \frac{\delta m}{M} \mu R^{3} \omega^{4} e^{-3 i \phi} \\
& I_{\text {nospin }}^{42}=\frac{16}{63} i \sqrt{2 \pi} \mu(1-3 \eta) R^{4} \omega^{5} e^{-2 i \phi} \\
& I_{\text {nospin }}^{44}=-\frac{256}{9} i \sqrt{\frac{2 \pi}{7}} \mu(1-3 \eta) R^{4} \omega^{5} e^{-4 i \phi}
\end{aligned}
$$

where $R$ is the radial separation and $\omega=\dot{\phi}$ is the binary orbital frequency. Considering only the mass quadrupole terms in the linear momentum flux (i.e., the terms proportional to $S^{21} I^{22 *}, I^{31} I^{22 *}$, and $\left.I^{22} I^{33 *}\right)$, we obtain the well-known result valid at Newtonian order [47]:

$$
F^{(0)}=-i \frac{464}{105} \frac{\delta m}{M} \mu^{2} R^{5} \omega^{7} e^{i \phi} .
$$

Including the next-highest order moments in Eq. (19), we get

$$
F^{(1)}=-i \frac{11120}{1323} \frac{\delta m}{M} \mu^{2}(1-3 \eta) R^{7} \omega^{9} e^{i \phi} .
$$

While there may also be next-to-leading order contributions from a PN expansion of the multipole moments included in Eq. (17) that would show up in Eq. (39), we can effectively absorb those corrections into the $R$ variable, as will be described below. 

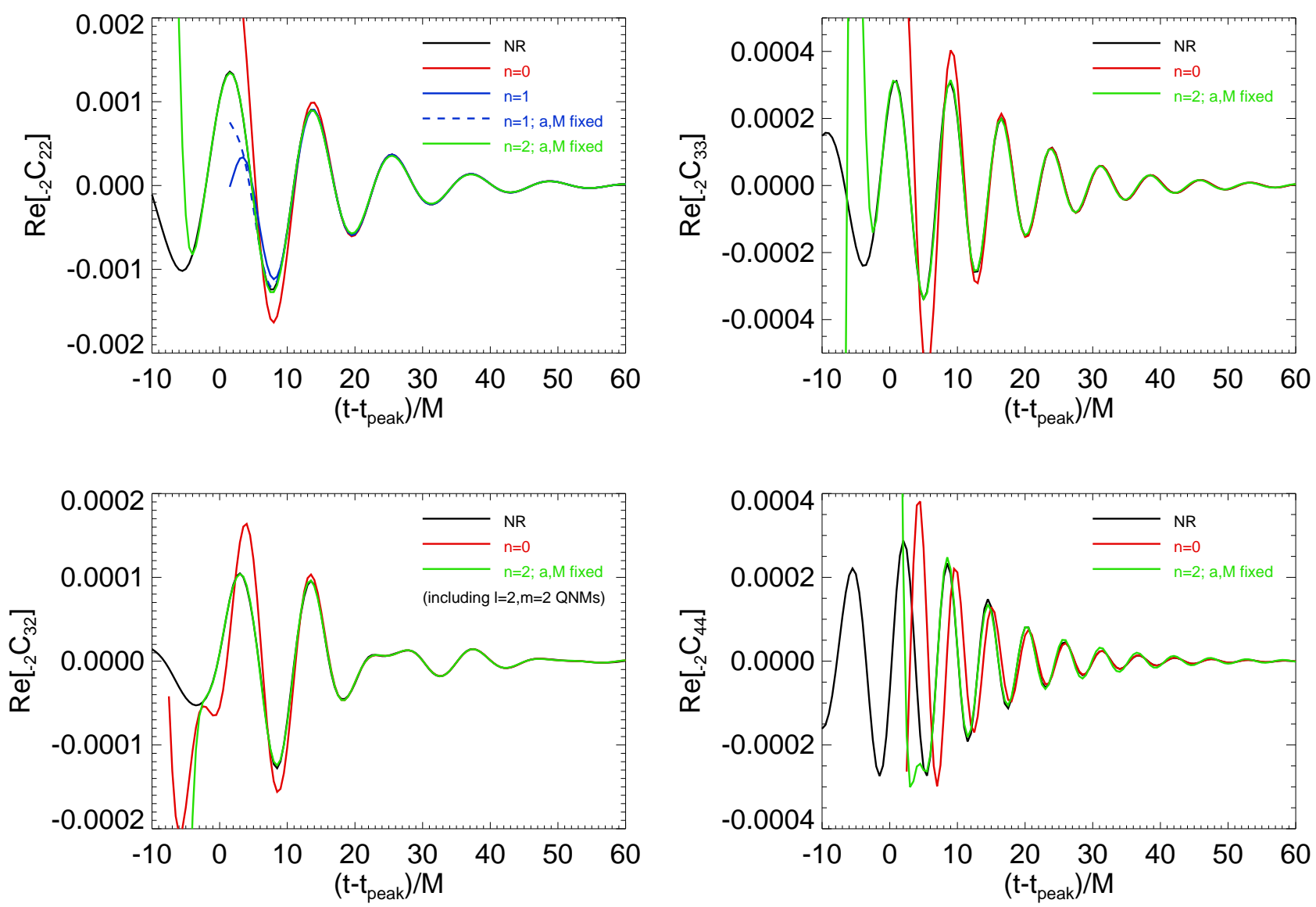

FIG. 5: Comparison of numerical and QNM waveforms for the $\mathrm{NE}_{00}^{2: 3}$ run. The dominant modes analyzed are ${ }_{-2} C_{22}$ (upper left), ${ }_{2} C_{33}$ (upper right), ${ }_{2} C_{32}$ (lower left), and ${ }_{-2} C_{44}$ (lower right). Note that the ${ }_{2} C_{32}$ waveform includes contributions from the $\ell=2, m=2$ modes as well. We denote with $t_{\text {peak }}$ the time of the peak of $I^{22}$.
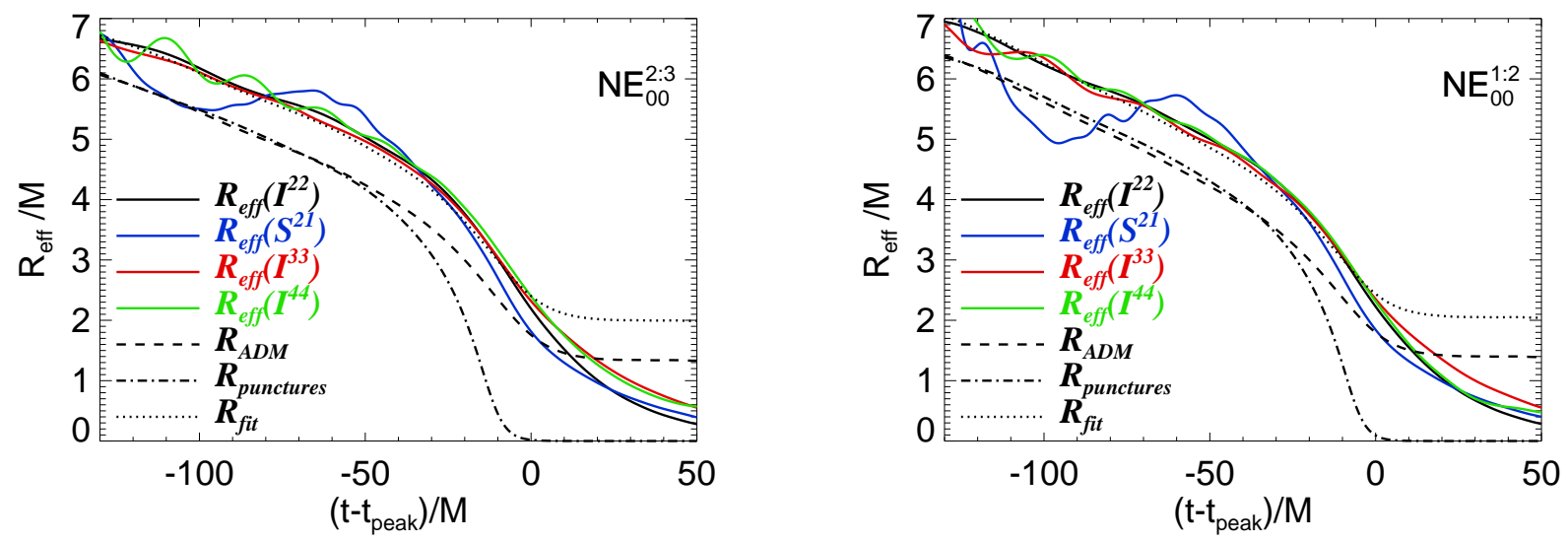

FIG. 6: Effective radius for different modes, derived from Eqs. (35), 37a - $37 \mathrm{~g})$. The close agreement for the $R_{\text {eff }}^{l m}$ suggests we can use a single effective radius $R_{\mathrm{eff}}(t)$ for the Newtonian expressions. We believe that the large oscillations in $R_{\mathrm{eff}}^{21}$ are due to initial eccentricity at early times. Also plotted is the ADM radius (dashed curves) derived from the orbital frequency via Eq. (41), the coordinate separation of the $\mathrm{BH}$ punctures (dot-dashed curves), and the empirical fit $R_{\mathrm{fit}}$ (dotted curves) obtained by shifting $R_{\mathrm{ADM}}$ by 0.65 . The results correspond to the $\mathrm{NE}_{00}^{2: 3}$ (left panel) and $\mathrm{NE}_{00}^{1: 2}$ (right panel) runs. We denote with $t_{\text {peak }}$ the time at which $I^{22}$ reaches its maximum. 
Combining Eqs. (38) and (39) we find the linear momentum flux scales like

$$
\begin{aligned}
\left|F^{(0)}+F^{(1)}\right| & \propto \frac{\delta m}{M} \mu^{2}\left[1+\frac{3475}{1827}(1-3 \eta) R^{2} \omega^{2}\right] \\
& \approx \frac{3}{2} \frac{\delta m}{M} \mu^{2}(1-0.9 \eta),
\end{aligned}
$$

which is remarkably similar to the result found in Ref. [9]. Here we have used $R^{2} \omega^{2} \approx 0.23-0.25$ at the peak of the energy flux, which seems to be quite robust for a range of mass ratios. However, the extremely close agreement with Ref. [9] is probably to some degree a coincidence, since this simple Newtonian formula does not include any details of the phase relations between different modes, which become especially important during the transition from inspiral to ringdown (see Sec. VIB below). Since Eq. (40) really only applies to the inspiral portion, if anything, it should be a predictor of how the peak recoil velocity scales. This is not necessarily the same as the final recoil, since we find that more extreme-mass-ratio $\mathrm{BH}$ binaries have a relatively smaller anti-kick, which should also play an important role in the scaling relation of Ref. [9].

If we compute the above multipole moments (37a)(37g) using $\omega$ as given by Eq. (35) and $R$ as obtained from the puncture trajectories, we do not find a very good agreement with the numerical results. This is not surprising since there is no reason to believe that the Newtonian approximation should work well all along the inspiral phase. We should expect that higher-order PN cor- rections become important as we approach the merger. Furthermore, $R$ is a coordinate-dependent quantity, and thus does not necessarily have the same meaning in a PN expression as in NR. Since our scope is limited to a diagnostic of the NR results, and not to a precise comparison with $\mathrm{PN}$ calculations, instead of including $\mathrm{PN}$ corrections in Eqs. (37)- (39), we investigate whether by properly scaling the Newtonian expressions we can get a better agreement until the merger. We can also think of this normalization as a way of resumming the PN expansion.

Quite interestingly, if we compute the amplitudes $\left|I^{\ell m}\right|$ or $\left|S^{\ell m}\right|$ from the numerical data, and the angular frequency $\omega$ from Eq. (35), we find that the radii $R^{\ell m}$ which appear in the RHS of Eqs. (37a)-(37g) are rather independent of the multipole moments $\ell$ and $m$, as Fig. 6 shows. We denote the radii $R^{\ell m}$ computed numerically as effective radii $R_{\mathrm{eff}}^{\ell m}$. The close agreement between the frequencies (see Fig. 2) and effective radii for each mode suggests we can use the Newtonian expressions and a single $R_{\text {eff }}(t)$ and orbital frequency $\omega(t)$, e.g., $R_{\text {eff }}^{22}(t)$ and $\omega_{\mathrm{D}}^{I 22}$ for all modes with a high degree of accuracy for the entire inspiral phase and even during the transition to merger.

For comparison we also show in Fig. 6] the radius from the puncture trajectory (dot-dashed curves) and the radius computed using the Arnowitt-Deser-Misner transverse-traceless gauge (dashed curves), given as a function of frequency through $3 \mathrm{PN}$ order by 74

$R_{\mathrm{ADM}}=M^{1 / 3} \omega^{-2 / 3}\left[1+\omega^{2 / 3}\left(-1+\frac{\eta}{3}\right)+\omega^{4 / 3}\left(-\frac{1}{4}+\frac{9}{8} \eta+\frac{\eta^{2}}{9}\right)+\omega^{2}\left(-\frac{1}{4}-\frac{1625}{144} \eta+\frac{167}{192} \eta \pi^{2}-\frac{3}{2} \eta^{2}+\frac{2}{81} \eta^{3}\right)\right]$

Here we use the orbital frequency $\omega$ derived from the $I^{22}$ mode via Eqn. (35), giving a constant value during the RD phase when the orbital frequency is meaningless. Fig. 6] shows interesting agreement between $R_{\mathrm{ADM}}$ and the radius from the puncture trajectory, and a constant offset between $R_{\mathrm{ADM}}$ and $R_{\text {eff }}$. The latter is due to the fact that the amplitude of the multipole moments computed at leading Newtonian order does not reproduce the numerical relativity amplitude [68, 73], and higher order PN corrections need to be included. Motivated by this similarity between $R_{\mathrm{ADM}}$ and $R_{\text {eff }}$, we attempt to fit empirically the $R_{\text {eff }}$ curves in Fig. 6 by simply shifting $R_{\mathrm{ADM}}$ by 0.65 . The fit curve is included as a dotted curve in Fig. 6. As we accumulate longer and more accurate NR data for a wider range of $\eta$ values, and study possible analytic resummation of higher-order PN amplitude corrections, we should be able to work out a widely applicable amplitude-scaling factor to be included in leading-order analytic waveforms [7].

In the next section, we shall investigate how this simple eN model can be combined with a superposition of QNMs, as described in Sec. IVB giving a good representation of the NR results.

\section{B. Matching to ringdown}

We now match the inspiral and $\mathrm{RD}$ waveforms in a mode-by-mode fashion following the philosophy of the EOB approach [39]. Note this is not the same analysis of SectionIVB, where we fit the numerical data throughout the RD phase with a superposition of QNMs. Here we match the data at a single point at the transition from inspiral to RD and see how well it agrees with the rest of the RD phase. A similar attempt was followed in Ref. [47], where for simplicity the authors performed the match- 
ing to the Schwarzschild QNM frequencies, while we use the Kerr QNM frequencies and match to the fundamental QNM frequency and the first two overtones, as done in Ref. 73]. We obtain the QNM frequencies and decay times from Ref. 72] as a function of $a_{\mathrm{f}} / M_{\mathrm{f}}$ (taken from Table I above). For the fundamental and two overtone QNMs, we can match a given multipole mode by equating it and two time derivatives to a linear combination of QNMs.

We write

$$
I^{\ell m}(t)=A(t) e^{-i \phi(t)}=\sum_{n=0}^{\infty} A_{\ell m n} e^{-i \sigma_{\ell m n}\left(t-t_{\mathrm{match}}\right)},
$$

where the complex QNM frequencies are known functions of the final $\mathrm{BH}$ mass and spin, and we must solve for the complex amplitudes $A_{\ell m n}$. Matching three QNMs we get

$$
\begin{aligned}
I^{\ell m}\left(t_{\mathrm{match}}\right) & =\sum_{n=0}^{2} A_{\ell m n}, \\
\frac{d}{d t} I^{\ell m}\left(t_{\mathrm{match}}\right) & =-i \sum_{n=0}^{2} \sigma_{\ell m n} A_{\ell m n}, \\
\frac{d^{2}}{d t^{2}} I^{\ell m}\left(t_{\mathrm{match}}\right) & =-\sum_{n=0}^{2} \sigma_{\ell m n}^{2} A_{\ell m n},
\end{aligned}
$$

or as a simple matrix equation

$$
\left(\begin{array}{ccc}
1 & 1 & 1 \\
-i \sigma_{\ell m 0} & -i \sigma_{\ell m 1} & -i \sigma_{\ell m 2} \\
-\sigma_{\ell m 0}^{2} & -\sigma_{\ell m 1}^{2} & -\sigma_{\ell m 2}^{2}
\end{array}\right)\left(\begin{array}{c}
A_{\ell m 0} \\
A_{\ell m 1} \\
A_{\ell m 2}
\end{array}\right)=\left(\begin{array}{c}
I^{\ell m} \\
\dot{I}^{\ell m} \\
\ddot{I}^{\ell m}
\end{array}\right) .
$$

In Fig. 7 we compare the NR modes to the modes obtained by the effective Newtonian model described in Sec. VA until $t_{\text {match }}$ and by the superposition of three QNMs for $t>t_{\text {match }}$. During the inspiral, the different moments are calculated according to Eqs. (37a)-(37g), using a single $R_{\text {eff }}$ and $\omega_{\mathrm{D}}$ determined from the $I^{22}$ mode, with the exception of the $S^{21}$ mode, where we instead use the higher frequency $\omega_{\mathrm{D}}^{S 21}$ (but same $R_{\text {eff }}$ ). We treat $t_{\text {match }}$ as a free parameter: if we stop the inspiral too early, the eN mode amplitudes are still growing, so the sudden transition to decaying RD modes prematurely reduces them. On the other hand, if the inspiral is continued too long, we tend to lose the important phase shifts between the modes that only begin during the transition to RD. This is particularly evident in the $I^{44}$ mode, which undergoes an unexplained phase-shift around the transition to RD, and also decays at somewhat different rate than is predicted from QNM theory (see above, Sec. IVB). Motivated by the results of Sec. IVB notably by the fact that a superposition of three QNMs can fit very well the NR waveforms starting from the peak of the energy flux, we choose as best-matching point the peak of the energy flux.

Having shown a reasonably close match for each of the radiative multipoles between the effective Newtonian model and the numerical data, it stands to reason that the total recoil calculated with this model should agree as well. This is shown in Fig. 8, where we have also varied the matching point around $t_{\text {peak }}$. We first note the close agreement between the eN models with varying $t_{\text {match }}$, suggesting the inspiral-to-ringdown matching method described above is relatively robust. Not surprisingly, since the individual modes agree, we also find reasonable agreement between the $\mathrm{NR}$ data and the eN predictions for the recoil.

However, this agreement may be partially fortuitous, since the eN model cannot predict the mode phase shifts around $t=t_{\text {peak }}$, most notably that of the $I^{44}$ mode described above. In SectionVIB below, we will examine this phasing in greater detail and show how it affects the overall kick. At this point, we unfortunately do not have a clear understanding of the underlying cause of the phase shift, but it may well be related to the slightly different times of transition from inspiral to ringdown for the different modes. Preliminary results also suggest that this de-phasing effect is reduced in more extreme-massratio systems, as we shall see in Appendix A.

\section{ANATOMY OF THE KICK}

In the above Sections, we have laid the groundwork for a multipolar analysis of the gravitational recoil, describing the momentum flux as a combination of radiative multipole modes. Along with the psuedo-analytic models for the inspiral and ringdown phases, we can now give a detailed description of the "anatomy" of the kick, namely the way the different modes combine to produce a peak recoil velocity, followed by a characteristic anti-kick and then asymptotic approach to the final value of the $\mathrm{BH}$ recoil.

\section{A. Contribution from different moments}

In Sec. III we showed how the radiative multipole moments contribute to the linear momentum flux through the integral of the $\Psi_{4}$ scalar [Eqs. (10), (12)]. Here, we want to determine exactly which modes we need to include in the multipole expansion Eq. (13) to get a good representation of the full recoil, and which are the pairs of modes in Eq. (21) that contribute most.

By including only a select choice of terms in the $\psi_{4}$ expansion Eq. (10), we can calculate the linear momentum flux by direct integration of Eq. (12) and compare it with the predictions of Eqs. (17)-(21), in each case including only the appropriate moments. This is a good way of double-checking those lengthy equations term-by-term, and in practice we find excellent agreement, limited only by the numerical accuracy of the simulations. Similarly, we can use this method of truncated expansion to determine which modes are necessary for calculating the recoil up to a given accuracy. The results of using higher and 

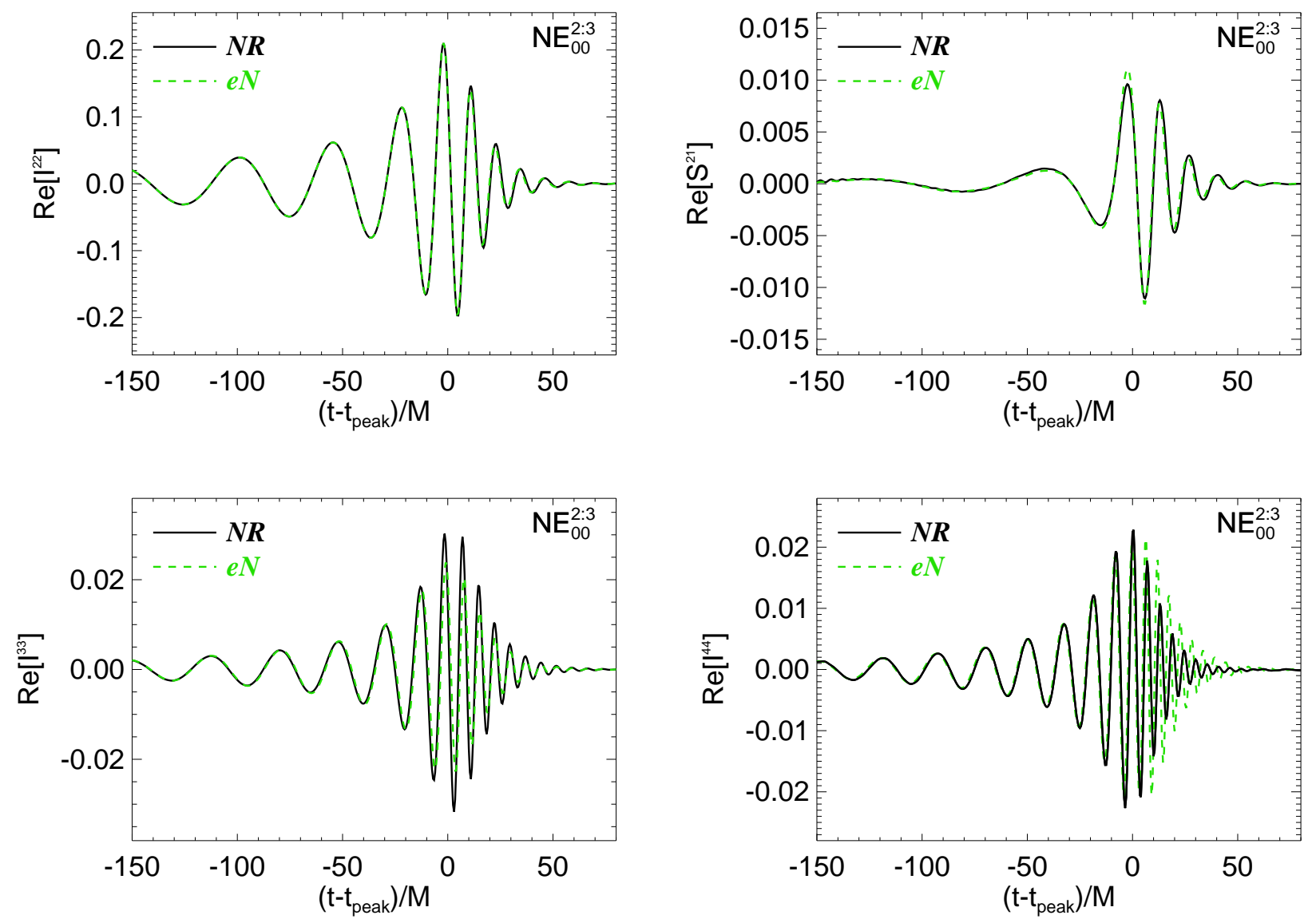

FIG. 7: Comparison of the effective Newtonian and NR radiative modes during inspiral, merger and RD phases. The data refer to the $\mathrm{NE}_{00}^{1: 2}$ run. We denote with $t_{\text {peak }}$ the time at which $I^{22}$ reaches its maximum.

higher order multipolar moments are shown in Figs. 9 and 10 for the $\mathrm{NE}_{00}^{2: 3}$ and $\mathrm{NE}_{00}^{1: 2}$ runs, respectively.

In the left panels of Figs. 9 and 10 we show with a solid curve the exact recoil velocity from Eq. (12), with a dashed curve the contribution from terms up to $\ell=4$, i.e., those obtained from Eq. (17) and (19), and with a dotted curve the contribution from just the three leading terms in Eq. (21), valid for non-precessing BHs with kicks in the orbital plane. We conclude that the linear momentum flux is dominated by the $I^{33} I^{22 *}, I^{33} I^{44 *}$, and $S^{21} I^{22 *}$ terms, which combine to produce the primary kick and anti-kick agreeing with the exact result within $\lesssim 10 \%$ throughout the entire merger. Note that the flux from the $S^{32} I^{33 *}$ term, while not insignificant in Fig. 4. contributes almost nothing to the net recoil velocity. This is largely due to phase relations between the various modes during the transition from inspiral to ringdown, described below in Sec. VIB.

In the right panels of Figs. 9 and 10 we show the difference between the calculation obtained including terms up to $\ell=3,4,5,6$, and the exact result. It seems clear that we need modes up to and including $\ell=4$ to get an accurate estimate of the recoil velocity. For more extreme mass ratios, higher-order moments become relatively more important, but remain strongly subdominant to the $\ell \leq 4$ modes $[11,75]$.

To understand more clearly the relative contributions of the different modes to the total recoil, we will include analysis of a few more simulations including nonprecessing spins. As mentioned above in Sec. [II A nonprecessing spins do not introduce any additional moments compared to the non-spinning simulations, but simply modify the relative amplitudes of the different modes in Eq. (21) by adding the spin terms. Thus, once we determine how the spins modify the individual modes, we can use the same analysis for the spinning and nonspinning cases.

Again equating the radiative multipole moments with the source moments, we get the leading order spin-orbit modifications to Eqs. (37a)-37g) [see Eqs. (3.14),(3.20) 


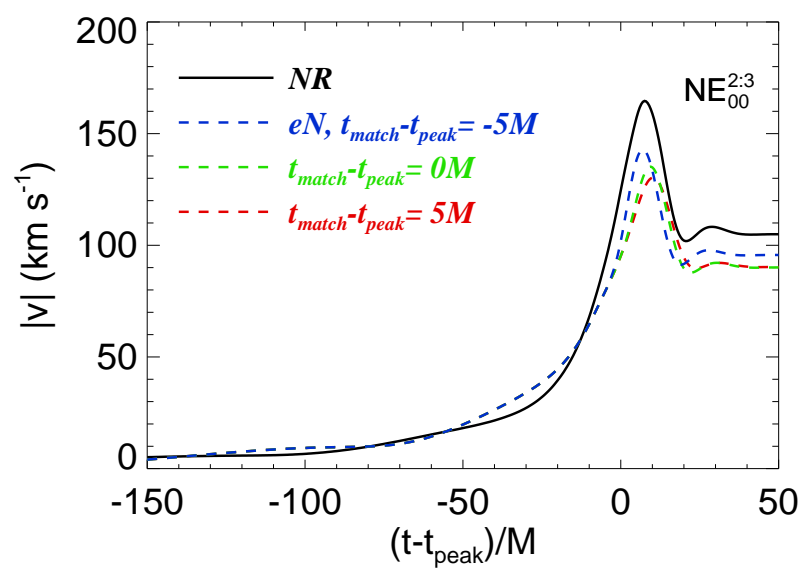

FIG. 8: Comparison of the effective Newtonian model and NR predictions for the recoil velocity for a range of inspiral-RD matching points. We denote with $t_{\text {peak }}$ the time at which $I^{22}$ reaches its maximum. The data refer to the $\mathrm{NE}_{00}^{2: 3}$ run.

in Ref. [44] and Eq. (5.5) in Ref. [76]]:

$$
\begin{aligned}
S_{\mathrm{SO}}^{21} & =-4 i \sqrt{\frac{2 \pi}{5}} \eta R \omega^{3} e^{-i \phi} \Delta^{z}, \\
I_{\mathrm{SO}}^{22} & =\frac{64}{3} i \sqrt{\frac{2 \pi}{5}} \eta R^{2} \omega^{4} e^{-2 i \phi} \xi^{z} \\
S_{\mathrm{SO}}^{32} & =-\frac{32}{3} \sqrt{\frac{2 \pi}{7}} \eta R^{2} \omega^{4} e^{-2 i \phi} \xi^{z}, \\
I_{\mathrm{SO}}^{31} & =-\frac{2}{3} \sqrt{\frac{\pi}{35}} \eta R^{3} \omega^{5} e^{-i \phi} \Sigma_{31}^{z}, \\
I_{\mathrm{SO}}^{33} & =54 \sqrt{\frac{\pi}{21}} \eta R^{3} \omega^{5} e^{-3 i \phi} \Sigma_{33}^{z},
\end{aligned}
$$

where we have introduced the spin vectors

$$
\begin{aligned}
& \Sigma_{31} \equiv \frac{11}{2} \frac{\delta m}{M} \mathbf{S}+\frac{1}{2}(11-39 \eta) \boldsymbol{\Delta}, \\
& \Sigma_{33} \equiv \frac{3}{2} \frac{\delta m}{M} \mathbf{S}+\frac{3}{2}(1-5 \eta) \boldsymbol{\Delta} .
\end{aligned}
$$

In all of the simulations considered here, the dimensionless spins are equal $\left(\left|a_{1}\right| / m_{1}=\left|a_{2}\right| / m_{2}\right)$ and point in opposite directions, $\xi^{z}=0$, so for the leading-order terms in Eqn. (21) we are left only with the modifications of $S^{21}$ and $I^{33}$, due to $\Delta^{z}$ and $\Sigma_{33}^{z}$, respectively. Then Eqs. (37) and (45) give the linear momentum flux during the inspiral for each of the first three dominant terms in Eq. (21):

$$
\begin{aligned}
& F_{\text {insp }}^{21,22}=\frac{16}{45} i \frac{\mu^{2}}{M} R^{3} \omega^{6}\left(2 \delta m R^{2} \omega+3 \Delta^{z}\right) e^{i \phi}, \\
& F_{\text {insp }}^{22,33}=-\frac{36}{7} i \frac{\mu^{2}}{M} R^{5} \omega^{7}\left(\delta m+\omega \Sigma_{33}^{z}\right) e^{i \phi}, \\
& F_{\text {insp }}^{33,44}=-\frac{64}{7} i \frac{\mu^{2}}{M}(1-3 \eta) R^{7} \omega^{9}\left(\delta m+\omega \Sigma_{33}^{z}\right) e^{i \phi} .
\end{aligned}
$$

While these flux formulae contain terms of various orders in $\omega$, we expect that the effective Newtonian scaling of $R$ ensures that we are including all relevant PN terms, at least in the cases where the $\delta m$ terms dominate over the spin corrections. When the spin terms begin to dominate, we find that it becomes more difficult to use a single effective $R$ for all modes. This can be seen in Fig. 11] which plots $R_{\text {eff }}$ as in Fig. [6 but for the $\mathrm{NE}_{-+}^{2: 3}$ run, where the $\Delta^{z}$ and $\delta m$ terms in Eq. (47a) are comparable, making it difficult to derive a reasonable $R_{\text {eff }}\left(S^{21}\right)$.

Even for non-spinning runs, in order to get reasonable agreement with the NR data, we find that one must be careful towards the end of the inspiral to distinguish between $\omega_{\mathrm{D}}^{I 22}$ and $\omega_{\mathrm{D}}^{S 21}$ in Eq. (47a):

$F_{\text {insp }}^{21,22} \propto\left(\mu^{2} / M\right) R^{3}\left(\omega_{\mathrm{D}}^{I 22}\right)^{3}\left(\omega_{\mathrm{D}}^{S 21}\right)^{3}\left(2 \delta m R^{2} \omega_{\mathrm{D}}^{S 21}+3 \Delta^{z}\right)$.

The amplitudes of these fluxes are plotted in Fig. 12 for the four runs $\mathrm{NE}_{-+}^{2: 3}, \mathrm{NE}_{+-}^{2: 3}, \mathrm{NE}_{00}^{2: 3}$, and $\mathrm{EQ}_{+-}$. As 

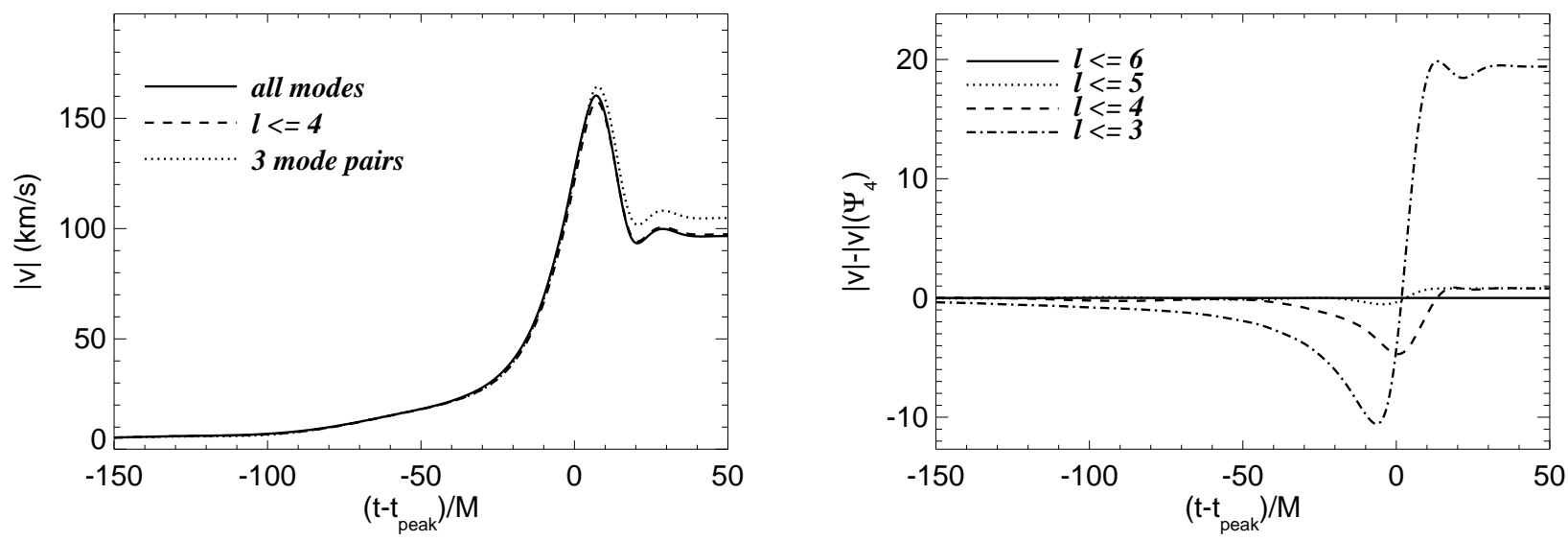

FIG. 9: In the left panel we show the net recoil kick, integrated from the linear momentum flux via Eq. (12) (solid curve), from all modes with $\ell \leq 4$ (dashed curve) and also limiting the modal composition of $\Psi_{4}$ to just the three dominant mode pairs in Eq. (21) (dotted curve). In the right panel we show the difference between the exact result and the $\Psi_{4}$ expansion Eq. (10), limited to $\ell \leq 3,4,5,6$. The data refer to the $\mathrm{NE}_{00}^{2: 3}$ run. We denote with $t_{\text {peak }}$ the time at which $I^{22}$ reaches its maximum.
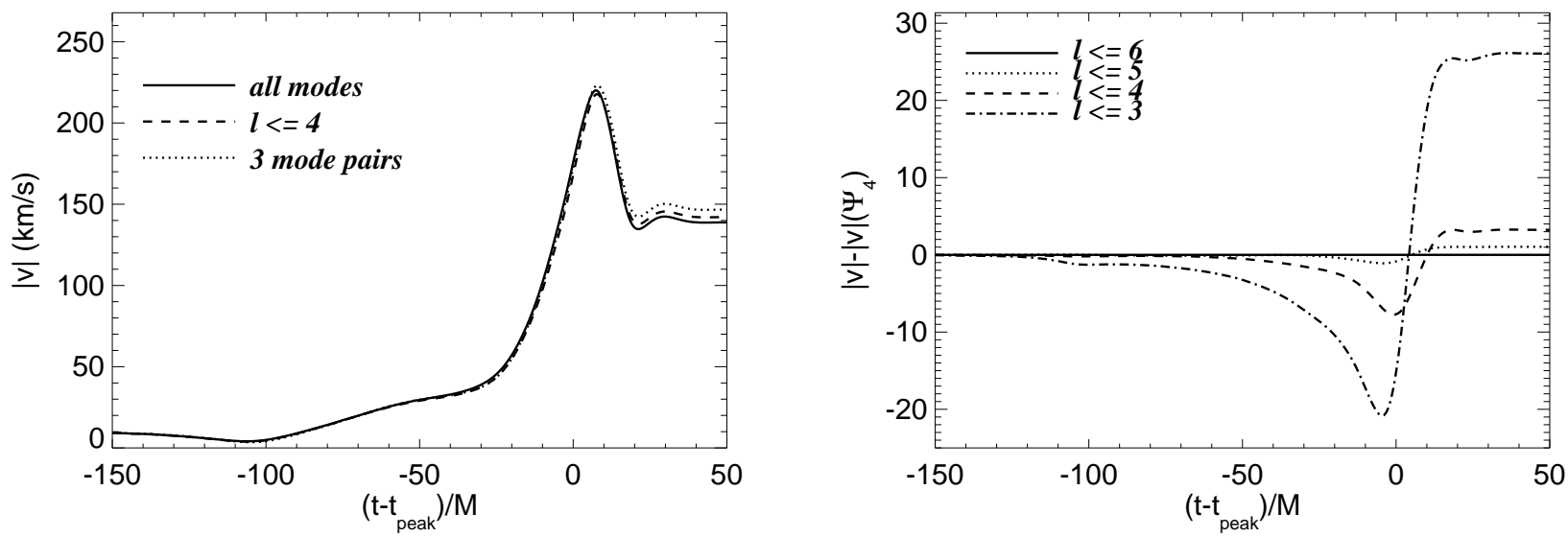

FIG. 10: Same as Fig. 9] but for the $\mathrm{NE}_{00}^{1: 2}$ run.

seen in Table I, the $\mathrm{NE}_{-+}^{2: 3}$ run has $\Delta^{z}=0.2 M^{2}$, while the $\mathrm{NE}_{+-}^{2: 3}$ run has $\Delta^{z}=-0.2 M^{2}$, respectively adding destructively and constructively with the $\delta m$ term in Eq. 47a). This difference is clearly seen in the blue curves in the top two panels of Fig. 12. Also notable in these plots is the somewhat smaller difference in the amplitudes of $F^{22,33}$, due to a similar effect from the constructive/destructive additions of $\delta m$ and $\Sigma_{33}^{z}$ in Eq. 47b. As we see in Fig. 12. $\mathrm{NE}_{00}^{2: 3}$ appears to be the average of $\mathrm{NE}_{+-}^{2: 3}$ and $\mathrm{NE}_{-+}^{2: 3}$, while the flux from $\mathrm{EQ}_{+-}$is strongly suppressed due to the $\delta m=0$ terms in Eq. (47), leaving only the flux from the terms proportional to $\Delta^{z}=-0.2 M^{2}$ and $\Sigma_{33}^{z}=0.075$. However, as noted above, when the spin terms dominate the flux, as in the case of equal-mass BHs, the eN model with a single $R_{\text {eff }}$ begins to break down. Yet even in this situation, Eqs. (47a)- 47c still have qualitative (if not quantitative) predictive value, including the relative phases between the different mode-pair fluxes during the inspiral.

In each panel of Fig. 12, we also plot with dashed lines the eN prediction for the various flux amplitudes. In almost all cases, the eN flux is quite close to the NR results up to about $10 M$ before $t_{\text {peak }}$, when the eN model begins to break down, especially for the spinning runs. The amplitude differences near the peaks are comparable to those seen in Fig. 7 for the $\mathrm{NE}_{00}^{2: 3}$ run. The notable exception is the $F^{21,22}$ flux from the $\mathrm{NE}_{-+}^{2: 3}$ and $\mathrm{EQ}_{+-}$ runs, where the spin terms dominate over the $\delta m$ terms.

\section{B. Transition to ringdown and the de-phasing of the multipole modes}

Since the flux vectors defined by Eq. (47) will not generally be co-linear, to understand the time evolution of the recoil velocity, we must first understand the phase 


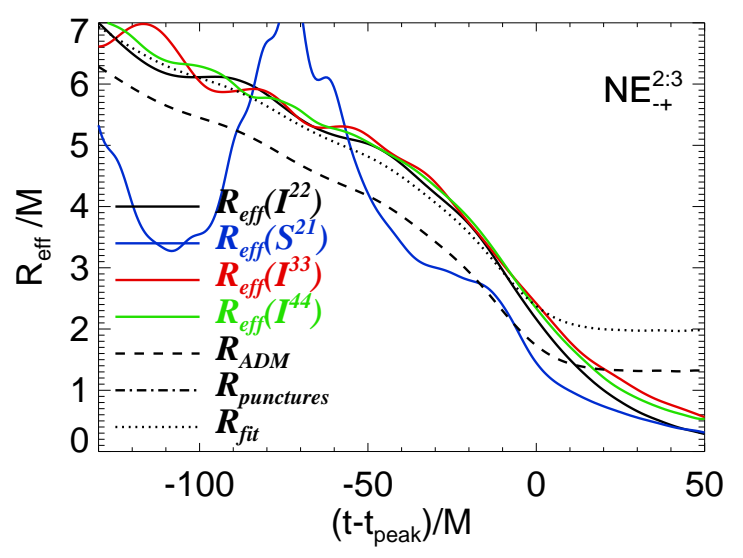

FIG. 11: $R_{\text {eff }}$ derived from different multipole modes, as in Fig. [6] for the $\mathrm{NE}_{-+}^{2: 3}$ run. The $S^{21}$ mode for this run has comparable contributions from $\delta m$ and $\Delta^{z}$, making it difficult to derive a reasonable $R_{\text {eff }}\left(S^{21}\right)$.

relations between the different modes. From Eqs. (37), (45), and (47), we see that during the inspiral phase, the individual moments and the resulting flux vectors evolve according to a single orbital phase $\phi$, with $F_{\text {insp }}^{21,22}$ pointing in the opposite direction to $F_{\text {insp }}^{22,33}$ and $F_{\text {insp }}^{33,44}$. However, as we can see from Fig. 2] as the binary evolves from inspiral to RD, the frequency (and thus phase) of the $S^{21}$ mode decouples from the other dominant modes. Upon closer inspection, we find that even the $I^{22}, I^{33}$ and $I^{44}$ modes deviate from each other enough to undergo a significant phase shift at the inspiral-RD transition.

To quantify these effects, we define the following phase differences:

$$
\begin{aligned}
\cos \psi^{2-3} & =\hat{\mathbf{F}}_{\text {insp }}^{21,22} \cdot \hat{\mathbf{F}}_{\text {insp }}^{22,33}, \\
\cos \psi^{2-4} & =\hat{\mathbf{F}}_{\text {insp }}^{21,22} \cdot \hat{\mathbf{F}}_{\text {insp }}^{33,44}, \\
\cos \psi^{3-4} & =\hat{\mathbf{F}}_{\text {insp }}^{22,33} \cdot \hat{\mathbf{F}}_{\text {insp }}^{33,44} .
\end{aligned}
$$

Here we use the notation $\psi^{m-m^{\prime}}$ to describe the phase difference between two complex flux vectors, where $m$ and $m^{\prime}$ correspond to the larger $m$-values of each mode pair that makes up the flux. These definitions are valid throughout the inspiral, merger, and ringdown phases. In the inspiral phase, we can see that for the unequalmass runs where $\delta m$ dominates with respect to the spin terms in Eqs. (47ad - 47c), we have

$$
\cos \psi_{\text {insp }}^{2-3}=\cos \psi_{\text {insp }}^{2-4}=-1, \quad \cos \psi_{\text {insp }}^{3-4}=1 .
$$

For the $\mathrm{EQ}_{+-}$run with $\delta m=0$, Eq. (47) predicts that all phases have $\cos \psi_{\text {insp }}=1$ during the inspiral (as shown in Table प. $\Delta^{z}$ and $\Sigma_{33}^{z}$ have opposite signs, so all the flux vectors in Eq. (47) are parallel). During the RD phase, using Eq. (42), we can approximate the flux vectors and phase evolution in terms of the fundamental QNM frequencies $\sigma_{\ell m 0}$ :

$$
\begin{aligned}
& F_{\mathrm{RD}}^{21,22} \simeq F_{\text {match }}^{21,22} \exp \left[-i\left(\sigma_{210}-\sigma_{220}^{*}\right)\left(t-t_{\mathrm{match}}\right)\right] \\
& F_{\mathrm{RD}}^{22,33} \simeq F_{\text {match }}^{22,33} \exp \left[-i\left(\sigma_{220}-\sigma_{330}^{*}\right)\left(t-t_{\text {match }}\right)\right] \\
& F_{\mathrm{RD}}^{33,44} \simeq F_{\text {match }}^{33,44} \exp \left[-i\left(\sigma_{330}-\sigma_{440}^{*}\right)\left(t-t_{\text {match }}\right)\right],
\end{aligned}
$$

where the $F_{\text {match }}^{\ell m, \ell^{\prime}}$ fluxes include complex phase information at the matching point. Taking the phase differences between these RD modes gives

$$
\begin{aligned}
& \cos \psi_{\mathrm{RD}}^{2-3} \simeq \cos \left[\left(\omega_{210}-2 \omega_{220}+\omega_{330}\right)\left(t-t_{\mathrm{match}}\right)+\Phi_{\text {match }}^{2-3}\right] \\
& \cos \psi_{\mathrm{RD}}^{2-4} \simeq \cos \left[\left(\omega_{210}-\omega_{220}-\omega_{330}+\omega_{440}\right)\left(t-t_{\text {match }}\right)+\Phi_{\text {match }}^{2-4}\right] \\
& \cos \psi_{\mathrm{RD}}^{3-4} \simeq \cos \left[\left(\omega_{220}-2 \omega_{330}+\omega_{440}\right)\left(t-t_{\text {match }}\right)+\Phi_{\text {match }}^{3-4}\right] .
\end{aligned}
$$

Here $\Phi_{\text {match }}$ is a phase offset determined at the transition from inspiral to ringdown. Quite interestingly, we find that for the range of final $\mathrm{BH}$ spin parameters $0.5 \leq a_{\mathrm{f}} / M_{\mathrm{f}} \leq 0.8$, the linear combinations of frequencies in Eqs. (52a) - (52C) vary by less than $\sim 5 \%$. Thus, if we compute the above expressions for the $\omega_{\operatorname{lm} 0}$ corre- sponding to $a_{\mathrm{f}} / M_{\mathrm{f}}=0.7$, we have [72]

$$
\begin{aligned}
& \cos \psi_{\mathrm{RD}}^{2-3} \simeq \cos \left[\frac{0.23}{M_{\mathrm{f}}}\left(t-t_{\text {match }}\right)+\Phi_{\text {match }}^{2-3}\right] \\
& \cos \psi_{\mathrm{RD}}^{2-4} \simeq \cos \left[\frac{0.22}{M_{\mathrm{f}}}\left(t-t_{\mathrm{match}}\right)+\Phi_{\text {match }}^{2-4}\right] \\
& \cos \psi_{\mathrm{RD}}^{3-4} \simeq \cos \left[\frac{0.012}{M_{\mathrm{f}}}\left(t-t_{\text {match }}\right)+\Phi_{\text {match }}^{3-4}\right]
\end{aligned}
$$



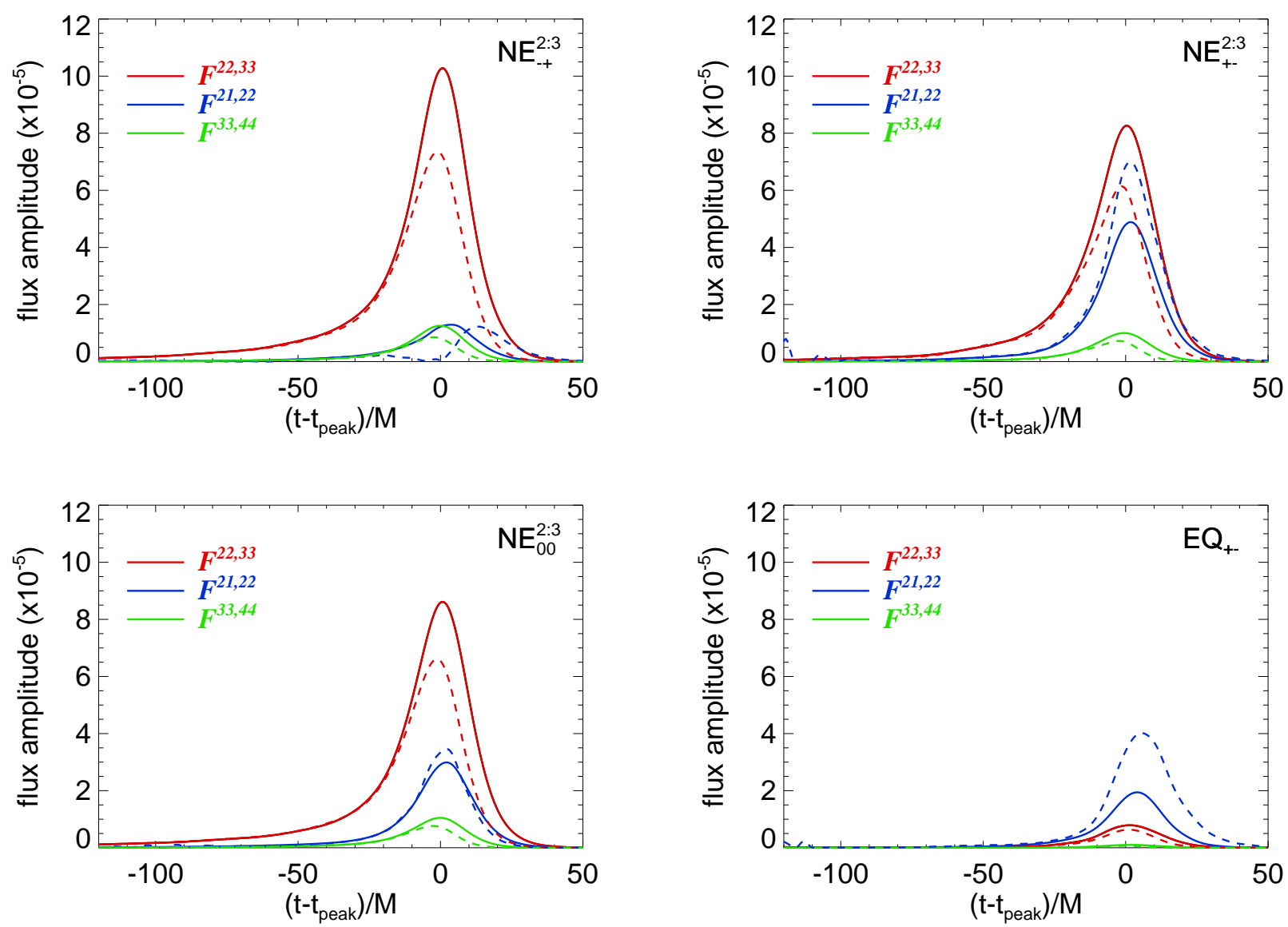

FIG. 12: Relative amplitudes of the dominant multipole mode-pairs in the linear momentum flux. Also shown in the dashed curves are the eN model predictions for the flux amplitudes. We denote with $t_{\text {peak }}$ the time at which $I^{22}$ reaches its maximum.

Even more intriguing, we find that for the unequalmass simulations described above, the phase relations during the inspiral and RD are almost identical, regardless of spin orientations. This can be seen clearly in Fig. 13 which plots $\cos \psi$ during inspiral, merger and $\mathrm{RD}$ for the different runs. The colinearity of the flux vectors is clear during the inspiral phase, and the sinusoidal oscillations of the phases during $\mathrm{RD}$ agree well with the analytic predictions (plotted in dashed curves in Fig. 131). Since the analytic models are most reliable during the inspiral and RD phases (but have more difficulty tracing the merger portion), we omit in Fig. 13 the transition region of $-10 M \leq\left(t-t_{\text {peak }}\right) \leq 10 M$. The analytic phase relations during inspiral are determined by Eq. (50) and during ringdown by Eqs. (53a)(53c). Here we use a $t_{\text {match }}$ (and corresponding $\Phi_{\text {match }}$ ) about $20 M$ after $t_{\text {peak }}$ to ensure that the multipole moments are truly dominated by the fundamental QNMs, and thus Eqs. (53a)-(53c) are valid. Note that the phase differences for $\mathrm{EQ}_{+-}$are particularly noisy since the amplitude of the $I^{33}$ moment is zero to leading order, and thus it is more difficult to extract a clear phase for that mode.
The feature that is most difficult to explain from an analytic model alone (and is thus omitted from the eN curves in Fig. 133) is the roughly 180-degree jump in phase between $F_{\text {insp }}^{22,33}$ and $F_{\text {insp }}^{33,44}$, beginning around $20 M$ before the peak. This appears to be a feature in all the unequalmass runs examined, but preliminary results suggest that is less significant (i.e., a smaller phase shift) for more extreme-mass ratio systems, as we shall discuss in Appendix A. We are not able to explain it with the additional RD overtone modes described in Sec. $\mathrm{VB}$ but using slightly different RD matching points for the different multipoles may help explain the issue.

\section{The anti-kick}

These flux amplitudes and phase relations can now be used to understand the amplitude of the kick and antikick, by which we mean the difference between the peak and the final recoil velocities (see Fig. 1 for an example). Throughout the inspiral phase, the amplitude and rotational frequency of the flux vectors in Eq. (47) are monotonically increasing, giving the familiar outward-spiraling 

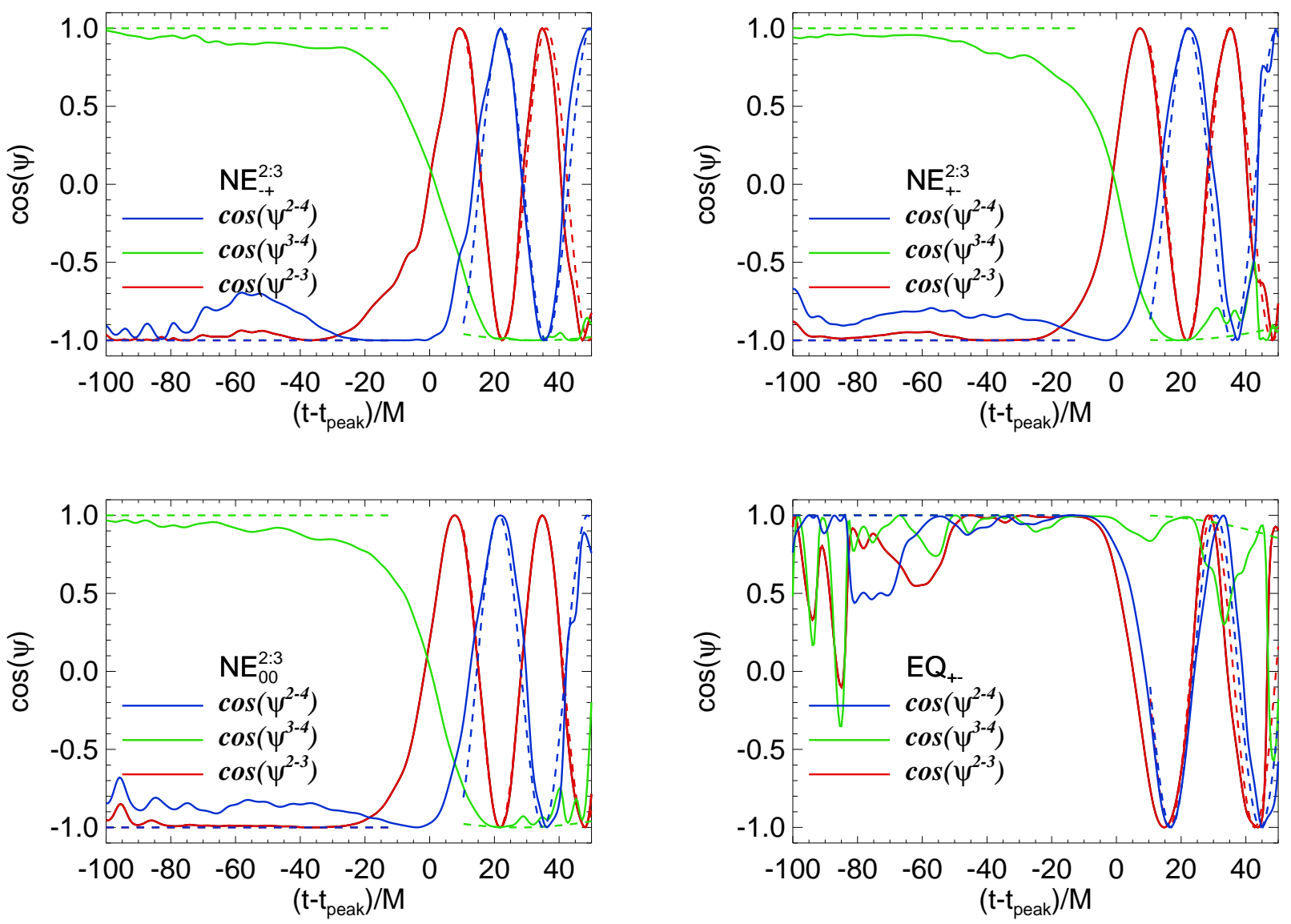

FIG. 13: Phase differences between different mode-pair flux vectors, as defined by Eqs. (49a)-(49c). The data refer to the $\mathrm{NE}_{+-}^{2: 3}$ (upper left panel), $\mathrm{NE}_{-+}^{2: 3}$ (upper right panel), $\mathrm{NE}_{00}^{2: 3}$ (lower left panel), and $\mathrm{EQ}+-$ (lower right panel) runs. The dashed curves are the eN model predictions of Eqs. (50), (53). We denote with $t_{\text {peak }}$ the time at which $I^{22}$ reaches its maximum.

trajectory for the velocity vector. Then, in the RD phase, the dominant frequencies are nearly constant while the amplitudes decay exponentially for each mode, giving an inward-spiral that decays like a damped harmonic oscillator around the final asymptotic recoil velocity.

These trajectories in velocity space can be seen in Fig. 14, along with the instantaneous flux vectors from the competing mode-pairs. Clearly, even small changes in the mass ratios and spins orientations of the BHs can give a rather diverse selection of velocity trajectories. Note in particular the difference between the $\mathrm{NE}_{-+}^{2: 3}$ run, dominated by the $F^{22,33}$ flux and a large anti-kick, and the $\mathrm{EQ}_{+-}$run, which in contrast is dominated by the $F^{21,22}$ flux. We find that the $\mathrm{EQ}_{+-}$run has no antikick, which can be explained by the slowly rotating flux vector that does not spiral back inwards, but rather drifts off slowly towards infinity during the ringdown. The difference between these two runs can be explained entirely by examining the real part of their fundamental QNM frequencies $\sigma_{\ell m 0}$, which in turn determine the rotation rates of the flux vectors in $\mathrm{Eq}$. (51): $\mathrm{EQ}_{+-}$is dominated by $\omega_{220}-\omega_{210}=0.08 / M_{\mathrm{f}}$, a much slower frequency than $\omega_{330}-\omega_{220}=0.31 / M_{\mathrm{f}}$, which causes the rapid inwardspiral of the $\mathrm{NE}_{-+}^{2: 3}$ run.

To calculate the recoil velocity, we must integrate the linear momentum flux vectors in time. (For the initial velocity vector, we integrate the post-Newtonian approximation for the momentum flux from $t=-\infty$ to the beginning of the numerical simulation [46]. This effectively sets the centers of the spiral curves in Fig. 14 to correspond to the origin in velocity space.) We can get a reasonable analytic approximation by using Eqs. (47) and (51) for the inspiral and RD phases, respectively. In the adiabatic inspiral, the complex recoil velocity $v=v_{x}+i v_{y}$ can be written as 

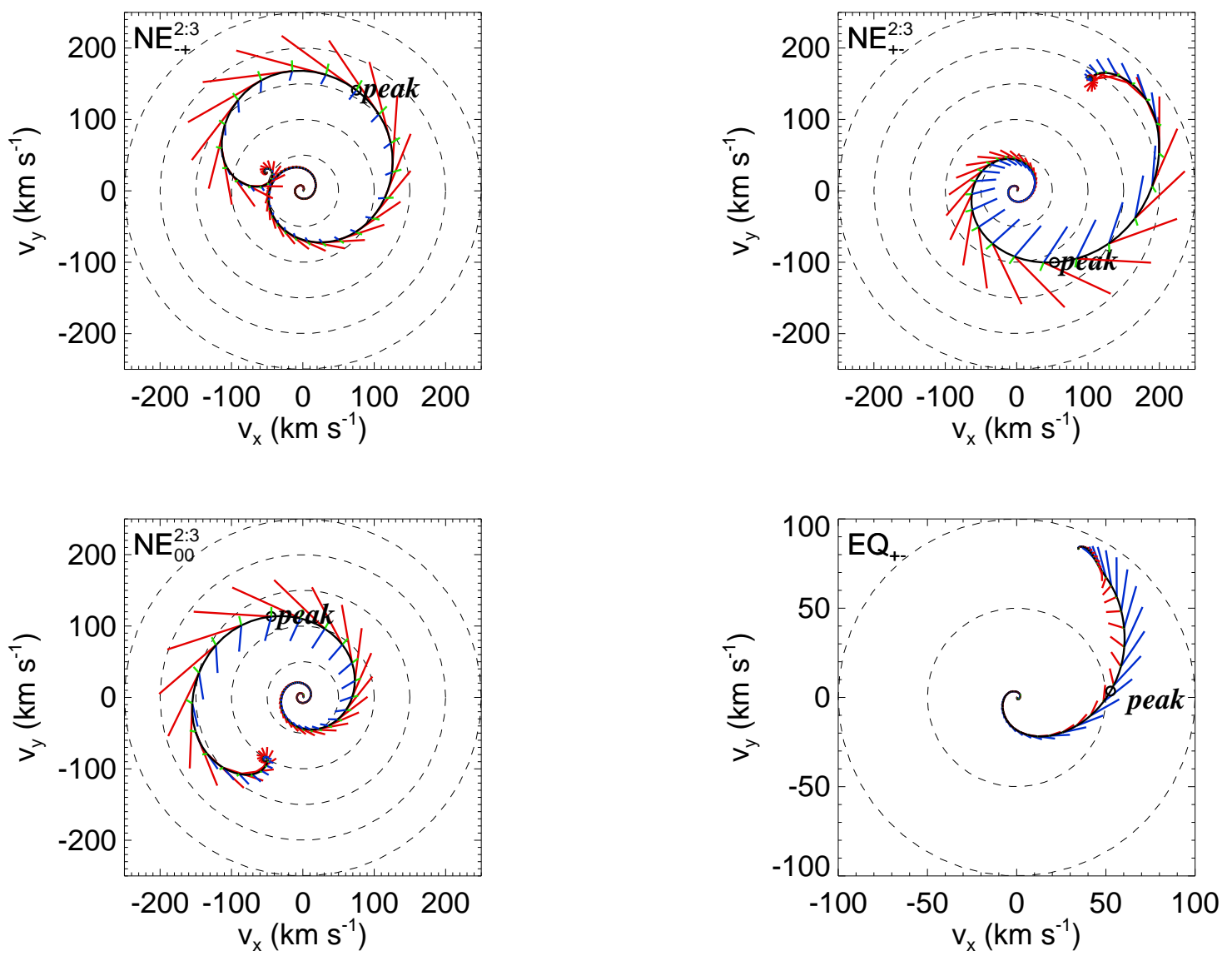

FIG. 14: The recoil velocity vector evolving in the $v_{x}-v_{y}$ plane (black solid curve), along with the flux vectors due to the three mode pairs at each time interval along the velocity trajectory (same color scheme as Fig. 12). The data refer to the $\mathrm{NE}_{+-}^{2: 3}$ (upper left panel), $\mathrm{NE}_{-+}^{2: 3}$ (upper right panel), $\mathrm{NE}_{00}^{2: 3}$ (lower left panel), and $\mathrm{EQ}_{+-}$(lower right panel) runs. We denote with the label peak the time at which $I^{22}$ reaches its maximum.

$$
v_{\text {insp }}=\int_{-\infty}^{t_{\text {match }}} F\left(t^{\prime}\right) d t^{\prime} \simeq \frac{1}{i \omega_{\text {match }}} F_{\text {match }}
$$

while for the RD portion we have

$$
\begin{aligned}
v_{\mathrm{RD}}(t)=\int_{t_{\mathrm{match}}}^{t} F\left(t^{\prime}\right) d t^{\prime} & \simeq \sum_{\ell m, \ell^{\prime} m^{\prime}} \frac{i F_{\mathrm{match}}^{\ell m, \ell^{\prime} m^{\prime}}}{\sigma_{\ell m 0}-\sigma_{\ell^{\prime} m^{\prime} 0}^{*}}\left[e^{-i\left(\sigma_{\ell m 0}-\sigma_{\ell^{\prime} m^{\prime} 0}^{*}\right)\left(t-t_{\mathrm{match}}\right)}-1\right], \\
v_{\mathrm{f}} \equiv v_{\mathrm{RD}}(t \rightarrow \infty) & \simeq \sum_{\ell m, \ell^{\prime} m^{\prime}} \frac{-i}{\sigma_{\ell m 0}-\sigma_{\ell^{\prime} m^{\prime} 0}^{*}} F_{\mathrm{match}}^{\ell m, \ell^{\prime} m^{\prime}},
\end{aligned}
$$

summing the contributions from each pair of modes $\left(\ell m, \ell^{\prime} m^{\prime}\right)$. Then the total velocity in each of the dominant mode 
pairs is given by

$$
\begin{aligned}
& \int F^{21,22}\left(t^{\prime}\right) d t^{\prime}=\frac{16}{45} \frac{\mu^{2}}{M} R_{\text {match }}^{3} \omega_{\text {match }}^{5}\left(2 \delta m R_{\text {match }}^{2} \omega_{\text {match }}+3 \Delta^{z}\right)\left[1-\frac{i \omega_{\text {match }} e^{i \phi_{\text {match }}^{21,22}}}{\sigma_{210}-\sigma_{220}^{*}}\right], \\
& \int F^{22,33}\left(t^{\prime}\right) d t^{\prime}=-\frac{36}{7} \frac{\mu^{2}}{M} R_{\text {match }}^{5} \omega_{\text {match }}^{6}\left(\delta m+\omega_{\text {match }} \Sigma_{33}^{z}\right)\left[1-\frac{i \omega_{\text {match }} e^{i \phi_{\text {match }}^{22,33}}}{\sigma_{220}-\sigma_{330}^{*}}\right], \\
& \int F^{33,44}\left(t^{\prime}\right) d t^{\prime}=-\frac{64}{7} \frac{\mu^{2}}{M}(1-3 \eta) R_{\text {match }}^{7} \omega_{\text {match }}^{8}\left(\delta m+\omega_{\text {match }} \Sigma_{33}^{z}\right)\left[1-\frac{i \omega_{\text {match }} e^{i \phi_{\text {match }}^{33,44}}}{\sigma_{330}-\sigma_{440}^{*}}\right] .
\end{aligned}
$$

The phase $\phi_{\text {match }}^{21,22}$ is defined as the angle made between the flux vector $F^{21,22}$ and the velocity vector $\mathbf{v}$ at the beginning of the ringdown (with other phases $\phi_{\text {match }}^{22,33}, \phi_{\text {match }}^{33,44}$ defined analogously). Because of the anomalous phase shifts and departure from adiabaticity at the transition from inspiral to ringdown, these angles are difficult to predict with an independent analytic model, but can be calculated easily from plots like Fig. 14. However, the accuracy of Eq. (56) is limited both by the adiabaticity condition of Eq. (54) as well as the accuracy of the spinorbit corrections to the eN model (see Fig. 11). Therefore, in analyzing the anti-kick in terms of RD modes, we find it more useful simply to integrate Eq. (54) directly from the numerical data during the inspiral, and then attach the fundamental QNM terms from Eq. (55) at the matching point $t_{\text {match }}=t_{\text {peak }}$.

Given $v_{\text {match }}$ at the end of the inspiral, we can use this quasi-analytic approach to predict the maximum and final recoil velocities $\left(v_{\max }\right.$ and $v_{\mathrm{f}}$, respectively). These predictions are plotted as black dashed curves in Fig. 15. to be compared with the solid black curves of the exact NR results. Within this context, we define the anti-kick magnitude as

$$
f_{\mathrm{ak}} \equiv \frac{v_{\mathrm{f}}-v_{\max }}{v_{\max }}
$$

and the net ringdown contribution as

$$
f_{\mathrm{RD}} \equiv \frac{v_{\mathrm{f}}-v_{\mathrm{match}}}{v_{\text {match }}}
$$

where $v_{\max }$ and $v_{\mathrm{f}}$ are the (real-valued) velocity magnitudes calculated analytically from Eq. (55).

In the case of the $\mathrm{NE}_{-+}^{2: 3}$ run, where the recoil is almost entirely dominated by the $F^{22,33}$ flux, we find a large anti-kick with $f_{\mathrm{ak}}=-0.53$ and $f_{\mathrm{RD}}=-0.5$. On the other hand, for the $\mathrm{NE}_{+-}^{2: 3}$ run, as can be seen in Fig. 15. the net recoil velocity continues to increase after $t_{\text {match }}=$ $t_{\text {peak }}$ before turning around for a small anti-kick of $f_{\mathrm{ak}}=$ -0.11 . The total effect of the ringdown phase is actually to increase the recoil with $f_{\mathrm{RD}}=0.68$. An intermediate effect is seen for the $\mathrm{NE}_{00}^{2: 3}$ run, with $f_{\mathrm{ak}}=-0.28$ and $f_{\mathrm{RD}}=-0.04$. However, as seen above in Fig. 14, for the $\mathrm{EQ}_{+-}$run, we see no anti-kick, with $f_{\mathrm{ak}}=-0.01$ and $f_{\mathrm{RD}}=0.58$.
In general, we find the magnitude of the anti-kick is primarily dependent on the relative magnitudes of the $S^{21}$ and $I^{33}$ moments. When $S^{21}$ dominates (e.g., when $\delta m$ and $\Delta^{z}$ add constructively), the ringdown rotation is slow and there is a small anti-kick, whereas a dominant $I^{33}$ mode (e.g., large $\delta m$ or no spins) gives a rapidly rotating ringdown flux and thus a large anti-kick. Furthermore, from Eq. (47), we see that for non-spinning BHs, both the $S^{21}$ and $I^{33}$ modes share the same mass and frequency scaling, so the relative size of the anti-kick should be roughly independent of mass ratio (see Appendix A for a caveat).

We would like a more quantitative picture of how these flux vectors add constructively and destructively to give the total recoil velocity to support the analytic estimates presented above. Using $\mathbf{v}=\int \mathbf{F} d t$, we can write

$$
\frac{d}{d t}|\mathbf{v}|=\frac{d}{d t}(\hat{\mathbf{v}} \cdot \mathbf{v})=\hat{\mathbf{v}} \cdot \mathbf{F}
$$

where $\hat{\mathbf{v}} \cdot \hat{\mathbf{v}}=1$. Breaking $\mathbf{F}$ up into the contributions of the dominant modes as above, and then integrating in time gives

$$
\begin{aligned}
v^{21,22} & =\int \hat{\mathbf{v}} \cdot \mathbf{F}^{21,22} d t, \\
v^{22,33} & =\int \hat{\mathbf{v}} \cdot \mathbf{F}^{22,33} d t, \\
v^{33,44} & =\int \hat{\mathbf{v}} \cdot \mathbf{F}^{33,44} d t,
\end{aligned}
$$

which add linearly to give to total recoil velocity:

$$
|\mathbf{v}|=v^{21,22}+v^{22,33}+v^{33,44} .
$$

Note that with these definitions, the $v^{\ell m, \ell^{\prime} m^{\prime}}$ are all real, but can be positive or negative. These different velocities are plotted in Fig. 15, with the same color scheme as in Figs. 12 and 14, along with the total recoil velocity in solid black curves. Also shown in Fig. [15] is the velocity $v^{32,33}$ (dashed blue curves), defined analogously to Eq. (60a) for the $S^{32} I^{33 *}$ flux terms. The small contribution from this mode pair further justifies our focus on the more dominant pairs of Eq. (21) and Fig. 4.

In the $\mathrm{NE}_{--3}^{2: 3}$ run, where the modal analysis shows the $F^{21,22}$ and $F^{33,44}$ flux terms canceling out, we see that 

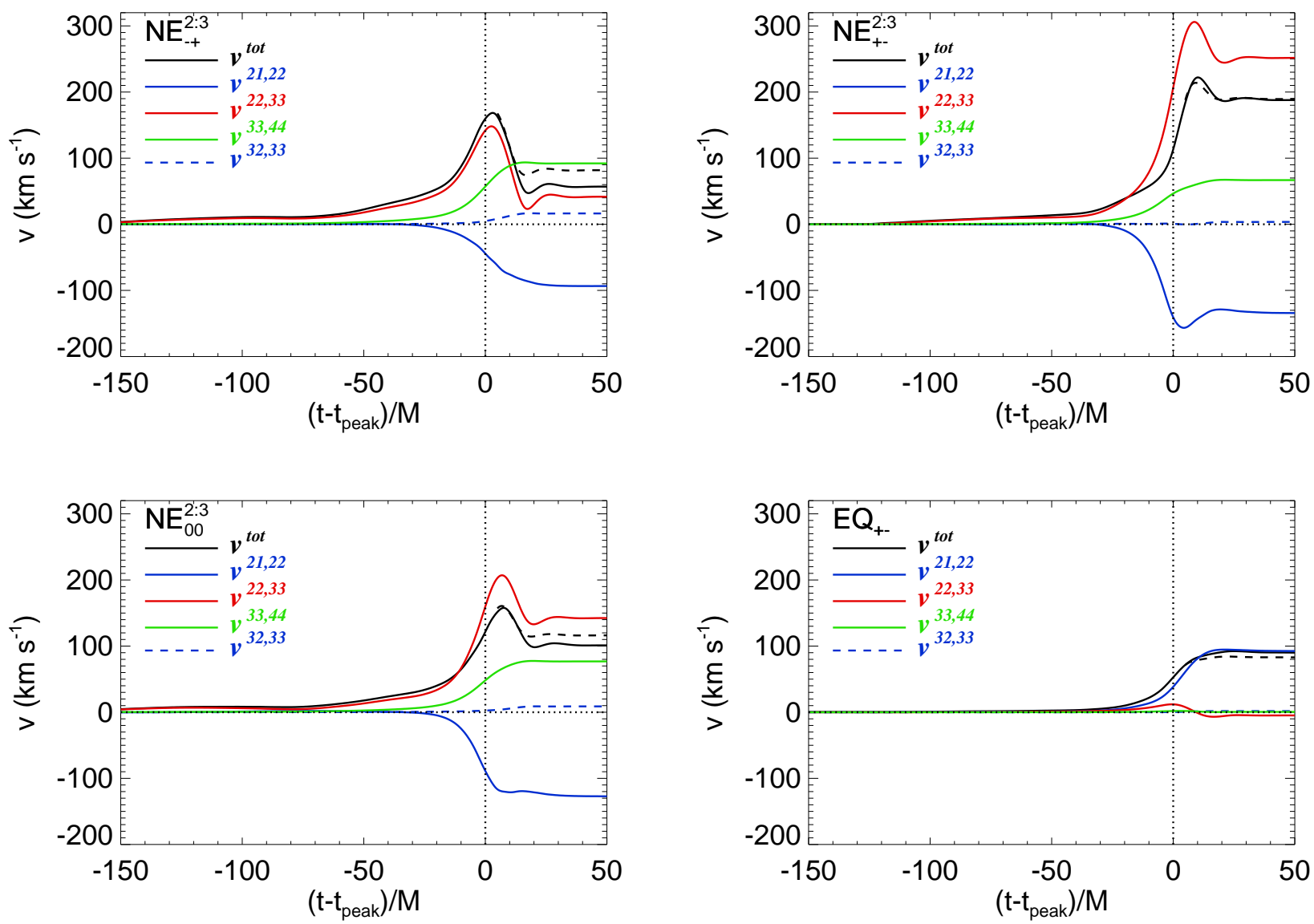

FIG. 15: Relative contributions to the total recoil velocity from the different multipole mode-pairs. $I^{22} I^{33 *}$ (red curve) is the dominant mode for unequal-mass binary systems, while $S^{21} I^{22 *}$ (blue curve) dominates for spinning, equal-mass binary systems. Also plotted is the contribution from the $S^{32} I^{33 *}$ flux terms (blue dashed curve), demonstrating its very small contribution to the total recoil velocity. For $t>t_{\text {match }}=t_{\text {peak }}$, we include the quasi-analytic prediction for $v_{\mathrm{RD}}(t)$ (black dashed curves), based on the fundamental RD modes from Eq. (55). The data refer to the $\mathrm{NE}_{+-}^{2: 3}$ (upper left panel), $\mathrm{NE}_{-+}^{2: 3}$ (upper right panel), $\mathrm{NE}_{00}^{2: 3}$ (lower left panel), and $\mathrm{EQ}_{+-}$(lower right panel) runs. We denote with $t_{\text {peak }}$ the time at which $I^{22}$ reaches its maximum.

the total recoil velocity (black curves in Fig. 15D) is almost entirely dominated by the $F^{22,33}$ flux (red curves). On the other hand, for the $\mathrm{NE}_{+-}^{2: 3}$ run, the $F^{21,22}$ flux is much stronger, adding destructively with the $F^{22,33}$ flux during the RD. This has the effect of both increasing the peak velocity and also decreasing the relative strength of the anti-kick, due to the slow rotation frequency of the $F^{21,22}$ flux during ringdown, as described above. As expected, the $\mathrm{NE}_{00}^{2: 3}$ run displays behavior intermediate between these two extremes. The $\mathrm{EQ}_{+-}$run, however, is entirely dominated by the $F^{21,22}$ flux, and thus experiences no anti-kick, but rather drifts off slowly in a nearly constant direction, as seen in the bottom-right panel of Fig. 14 .

\section{Application to non-planar kicks}

One of the most remarkable results from the recent renaissance in numerical relativity was the prediction of extremely large kicks from equal-mass BHs with spins pointing opposite to each other and normal to the orbital angular momentum, producing a recoil out of the orbital plane 13, 14, 15, 16]. While this configuration can produce recoils of nearly $4000 \mathrm{~km} / \mathrm{sec}$, the analogous non-precessing configuation $\left(\mathrm{EQ}_{+-}\right.$run in this paper) gives a kick of only $\sim 500 \mathrm{~km} / \mathrm{sec}$ in the case of maximal spin [10, 11, 12]. The multipole analysis tools developed above can be used for understanding and explaining this remarkable difference.

First, we should note that leading-order PN estimates of the linear momentum flux during inspiral suggest that the discrepancy should be less than a factor of two. For example, Eq. (3.31b) of Kidder [44] gives the spin-orbit contribution to the momentum flux for circular, Keplerian orbits as

$$
\mathbf{F}_{\mathrm{SO}}=\frac{16}{15} \mu^{2} M \frac{\omega^{2}}{R^{3}}[\hat{\mathbf{n}} \times \boldsymbol{\Delta}+(\hat{\mathbf{n}} \times \hat{\mathbf{v}})(\hat{\mathbf{v}} \cdot \boldsymbol{\Delta})]
$$

with $\hat{\mathbf{n}}$ and $\hat{\mathbf{v}}$ being the normalized separation and veloc- 
ity vectors, respectively. For spins parallel to the orbital angular momentum, the term in square brackets has magnitude $\Delta^{z}$, while for planar spins, it is $2 \Delta^{p} \sin \phi_{\Delta}$, where $\phi_{\Delta}$ is the angle between $\boldsymbol{\Delta}$ and $\mathbf{n}$, and $\Delta^{p}$ is the magnitude of $\boldsymbol{\Delta}$ in the orbital plane.

Not surprisingly, we get the exact same results from the multipole analysis of Eqs. (17), (18), (45), and one new multipole moment:

$$
S_{\mathrm{SO}}^{22}=4 i \sqrt{\frac{2 \pi}{5}} \eta R \omega^{3} e^{-i \phi}\left(\Delta^{x}-i \Delta^{y}\right)
$$

while on the other hand, the $S^{21}$ mode is zero for the planar spin configuration. Combining these equations, we get

$$
F_{x}+i F_{y} \approx \frac{1}{336 \pi}\left(-14 i S^{21} I^{22 *}\right)=\frac{16}{15} i \frac{\mu^{2}}{M} R^{3} \omega^{6} \Delta^{z} e^{i \phi}
$$

and using Eq. (18) we obtain

$$
\begin{aligned}
F_{z} \approx \frac{1}{336 \pi}\left[-28 \Im\left(I^{22} S^{22 *}\right)\right] & =\frac{32}{15} \frac{\mu^{2}}{M} R^{3} \omega^{6}\left(\Delta^{x} \sin \phi-\Delta^{y} \cos \phi\right) \\
& =\frac{32}{15} \frac{\mu^{2}}{M} R^{3} \omega^{6} \Delta^{p} \sin \phi_{\Delta}
\end{aligned}
$$

where $\phi$ is the orbital phase of the binary. So in both paradigms, we see that, when maximizing over $\sin \phi_{\Delta}$, the planar-spin orientation should result in a recoil twice as large as the parallel-spin case, leaving a factor of roughly 4 difference unexplained.

From Eqs. 64, 65 we see that the only relevant modes involved should be $I^{22}, S^{21}$, and $S^{22}$ (for these equal-mass systems the momentum flux is dominated by a single mode pair, responsible for $\gtrsim 95 \%$ of the final recoil value). In the left panel of Fig. [16 we plot the amplitude of $I^{22}$ from the EQ+- simulation, along with that of a planar-spin simulation EQplanar. All other binary parameters and the initial conditions are the same. Remarkably, the mass-quadrupole moments $I^{22}$ are nearly identical (and dominant) in both runs, and this suggests that the energy and angular momentum fluxes are the same [see Eqs. (31), (32)]. This is in fact quite reasonable since the total spin of the system is zero in both cases. However, we see in the right-hand panel of Fig. 16 that the peak amplitude of the $S^{22}$ mode is a factor of $\sim 2.5$ greater than that of the $S^{21}$ mode from the $\mathrm{EQ}_{\text {planar }}$ and $\mathrm{EQ}_{+-}$runs, respectively.

Yet Eqs. (45), (63) suggest that these two modes should have exactly the same magnitudes, at least during the inspiral phase, and presumably during the RD as well, since the RD amplitudes are completely determined by the mode amplitudes at the matching point. It appears from Fig. 16 that $S^{22}$ and $S^{21}$ do in fact have the same amplitude at early times, but the relatively noisy data and short duration of the simulations make it impossible to say for certain. If this is the case, one possible explanation for the sudden remarkable increase in the amplitude of $S^{22}$ might be mode-coupling with the dominant $I^{22}$ mode, as the inspiral phase begins to transition to the RD phase. This coupling is analogous to that of $S^{32}$ and $I^{22}$ described above in Sec. IVB an effect that is apparently only important between modes with the same $m$-number 72, 73]. We hope to address this question in the future with longer simulations to confirm the agreement at early times, as well as other spin configurations that should enhance specific multipole modes and may help identify other similar cases of mode amplification.

Lastly, from the ringdown contribution to the velocity [Eqs. (55), (56)], we can understand another difference between the planar- and parallel-spin orientations. Instead of having two different RD frequencies $\sigma_{210}$ and $\sigma_{220}$ combine to give a slowly rotating flux vector, for the planar-spin case, we have two identical RD frequencies for $I^{22}$ and $S^{22}$ in Eq. (65), giving precisely zero rotation to the RD flux. Furthermore, as the spin vector $\boldsymbol{\Delta}$ is precessing faster and faster in a positive direction around the orbital angular momentum vector, even during the inspiral the two modes $I^{22}$ and $S^{22}$ become nearly locked in phase, producing a relatively longduration burst of linear momentum flux in a single direction during the merger phase. Combined, these effects essentially straighten out the spiral curve in the lowerright panel of Fig. 14, providing another factor of $\sim 1.6$ of increased recoil velocity for planar spins.

In Fig. 17 we show the combination of the above effects. In the left panel, we plot the linear momentum flux from Eqs. (64), 65), showing the factor of two increase predicted by the Kidder formula and our Eqs. (17), (18), along with the factor of 2.5 increase in the amplitude of 

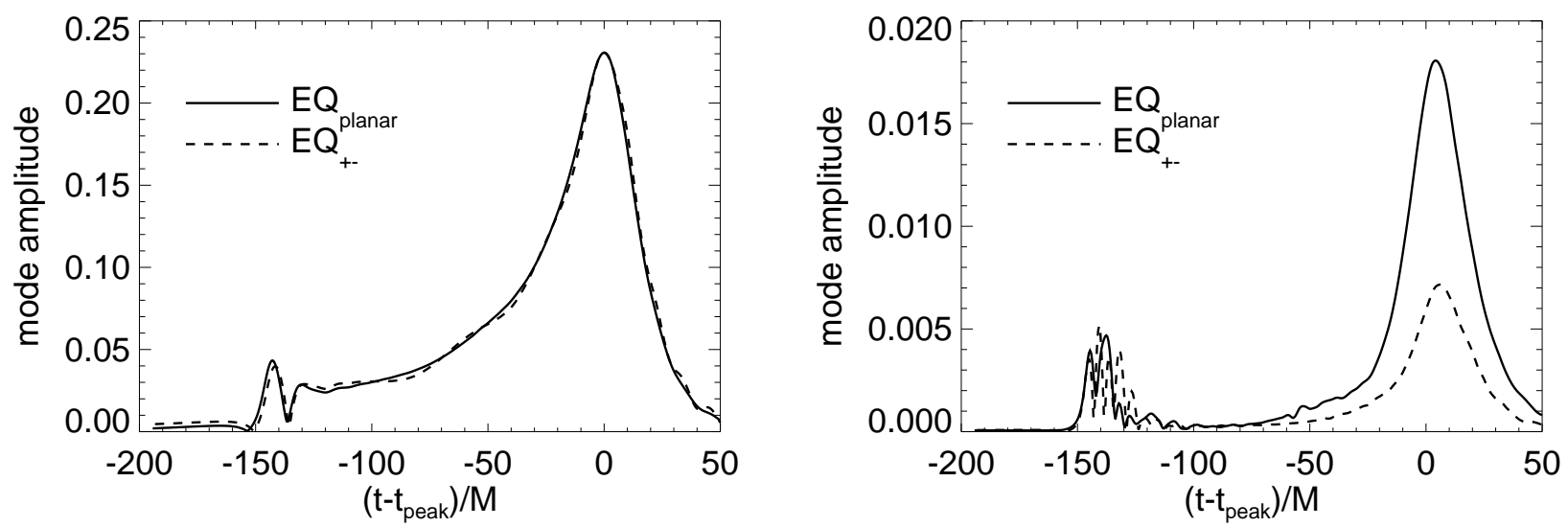

FIG. 16: left panel: Comparison of the multipole amplitudes $I^{22}$ for the two different equal-mass simulations: EQ planar $_{\text {(solid }}$

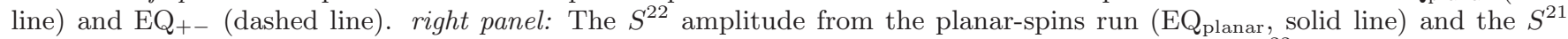
amplitude from the parallel-spins run $\left(\mathrm{EQ}_{+-}\right.$, dashed line). We denote with $t_{\text {peak }}$ the time at which $I^{22}$ reaches its maximum.
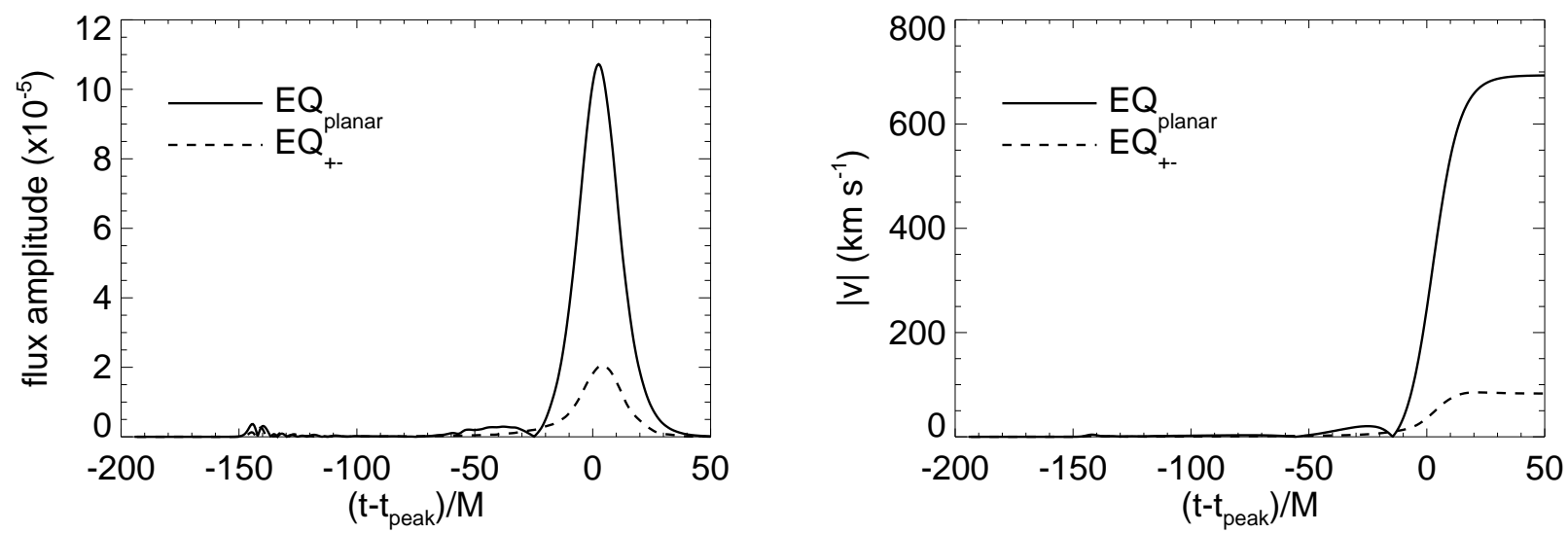

FIG. 17: left panel: Comparison of the linear momentum flux for the two different equal-mass simulations: EQplanar (solid line) and $\mathrm{EQ}_{+-}$(dashed line). right panel: The total recoil velocity from the planar-spins run (EQ $\mathrm{E}_{\text {planar }}$, solid line) and the parallel-spins run $\left(\mathrm{EQ}_{+-}\right.$, dashed line). We denote with $t_{\text {peak }}$ the time at which $I^{22}$ reaches its maximum.

$S^{22}$ relative to $S^{21}$. In the right panel, we plot the recoil velocity for both runs, which includes the effect of flux rotation during the merger and inspiral phases, accounting for another factor of $\sim 1.6$, giving a total discrepancy of $v\left(\mathrm{EQ}_{\text {planar }}\right) / v\left(\mathrm{EQ}_{+-}\right) \approx 2.5 \times 2 \times 1.6=8$.

\section{DISCUSSION}

In this paper we analysed several numerical simulations of binary $\mathrm{BH}$ coalescence, focusing on the physics of the recoil. We developed tools, based on the multipolar expansion [50, 51, 52, 53, 54], that can be used as a diagnostic of the numerical results, and understand how the recoil velocity evolves during the inspiral, merger, and ringdown phases of the coalescence.

We wrote explicit expressions for the linear momentum flux expressed in terms of radiative multipole moments through $\ell=4$, valid for generic spinning, precessing $\mathrm{BH}$ binary systems. We found that these formulae are sufficient to obtain the total recoil velocity with high accuracy. By comparing the amplitudes of the different multipole moments, we found that in the case of nonprecessing spins-and thus a recoil in the orbital planeonly three pairs of modes contribute to most of the linear momentum flux, notably $S^{21} I^{22 *}, I^{22} I^{33 *}$ and $I^{33} I^{44 *}$. Those modes account for the total recoil with an accuracy on the order of $\sim 5-10 \%$ throughout the simulations. (see Figs. 9, 10).

The way in which the contribution from these three pairs of modes builds up is not trivial, since not only the relative amplitudes, but especially, the relative phases are also quite important. We found that the relative phases between the three mode-pairs are nearly constant during 
the inspiral phase, but start diverging at the onset of the transition from inspiral to RD (see Fig. 13). The latetime evolution can be described reasonably well with analytic formula obtained expressing the mode-pairs in terms of fundamental QNMs of a Kerr BH. We showed that it is the relative magnitude of the current-quadrupole mode $S^{21}$ and the mass-octupole mode $I^{33}$, together with the differences of the QNM fundamental frequencies for each of the dominant modes, that determine the difference between the recoil at the peak of the linear momentum flux, and the final recoil velocity, i.e., the magnitude of the anti-kick.

With the final goal of improving analytic PN models, we also explored whether simple modifications of the Newtonian formula for the linear momentum flux allow us to match the numerical results all along the binary evolution. We found that, if we treat the binary radial separation in the Newtonian multipole modes (37a)-37e with an effective radius, which is computed from the numerical simulations assuming that each multipole mode is described by a dominant frequency (see Fig. 2), the leading Newtonian modes reproduce quite well the numerical ones (see Figs. 17 8) up to the end of the inspiral phase. We also found, confirming the results in Ref. [73], that a superposition of three QNMs can fit the numerical waveforms very well from the peak of the radiation through the RD phase.

The tools developed in this paper will be employed to improve current analytic predictions for the recoil velocity [46, 47] using PN analytic models [36] and the EOB approach 37, 38, 39, 40, 42, 77]. An accurate, fully analytic description of the recoil velocity can be adopted in fast Monte Carlo simulations to predict recoil distributions from BH mergers with uncertainties smaller than in Ref. [32]. Those recoil distributions can in turn be included in simulations of hierarchical merger models of supermassive BHs providing more robust predictions for LISA.

\section{Acknowledgments}

We thank Emanuele Berti for providing us with tabulated data for the Kerr QNM frequencies. We would like to thank the anonymous referee for their careful and constructive comments.

A.B. and J.D.S. acknowledge support from NSF grant PHYS-0603762, and A.B. was also supported by the Alfred P. Sloan Foundation. The work at Goddard was supported in part by NASA grant 05-BEFS-05-0044 and 06-BEFS06-19. The simulations were carried out using Project Columbia at the NASA Advanced Supercomputing Division (Ames Research Center) and at the NASA Center for Computational Sciences (Goddard Space Flight Center). B.J.K. was supported by the NASA Postdoctoral Program at the Oak Ridge Associated Universities. S.T.M. was supported in part by the Leon A. Herreid Graduate Fellowship.

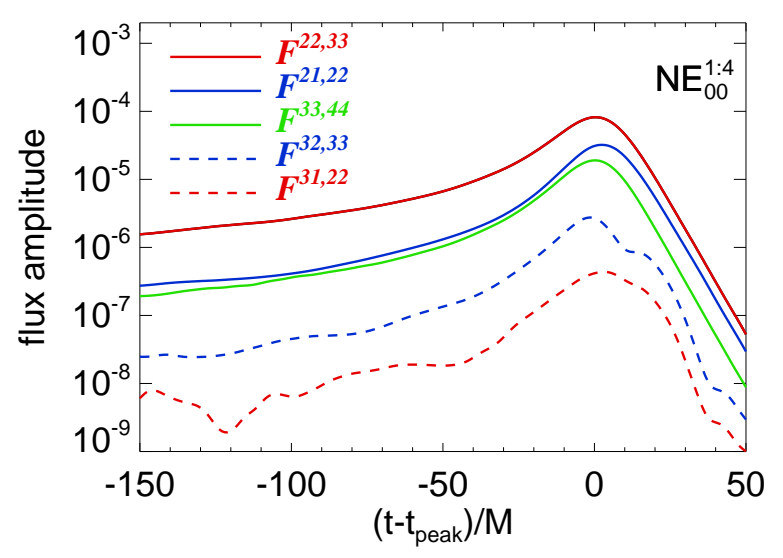

FIG. 18: Flux amplitudes from the $\mathrm{NE}_{00}^{1: 4}$ run, as in Fig. 4

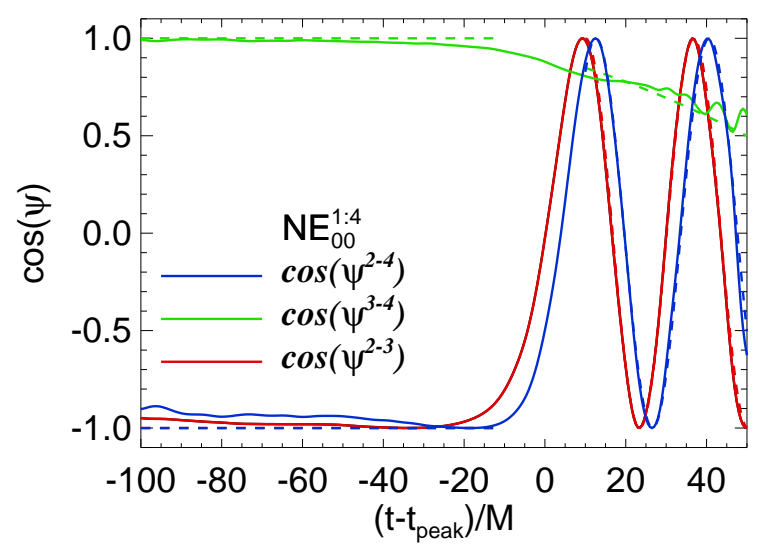

FIG. 19: Phase differences from the $\mathrm{NE}_{00}^{1: 4}$ run, as in Fig. 13

\section{APPENDIX A: RESULTS FROM 1:4 MASS RATIO}

In addition to the simulations presented in the main body of this paper, we have also recently analyzed a nonspinning system with mass ratio $1: 4(\eta=0.16)$. The results of this analysis are presented briefly in this appendix, as well as in Tables ПII (labeled appropriately as $\left.\mathrm{NE}_{00}^{1: 4}\right)$. More details can be found in Ref. 77].

In Fig. 18 we show the flux amplitudes from the different modes, as in Fig. 4 above. We find the relative amplitudes almost identical to those of the $\mathrm{NE}_{00}^{1: 2}$ run, with a slightly stronger contribution from the $I^{44}$ mode, as expected from Eq. (37g), which predicts a maximum in the $I^{44}$ amplitude for $\eta=0.167$.

In Fig. 19 we plot the phase relations between the different flux vectors, defined in Eqs. (49a)-(49c). As anticipated in Sec. VIB above, we find a smaller phase shift in $\psi^{3-4}$ at the transition from inspiral to ringdown for this more extreme mass-ratio system. The other phases appear to behave as expected. 


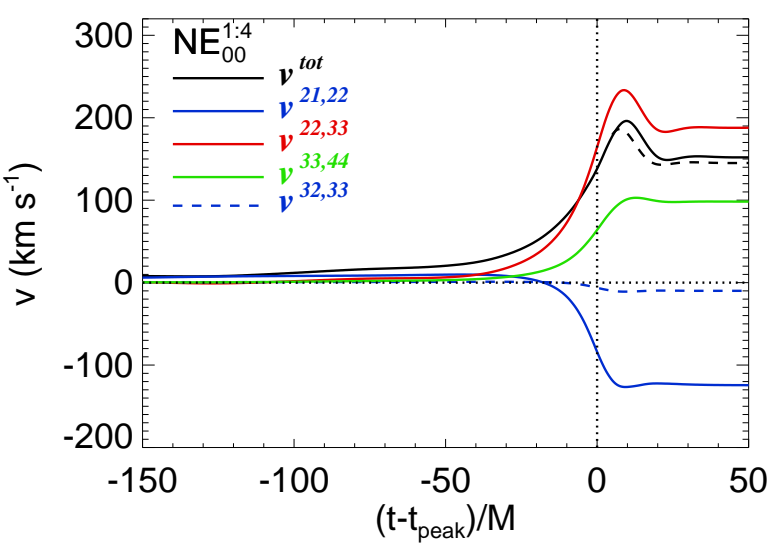

FIG. 20: Relative contributions to the total recoil velocity from the different multipole mode-pairs for the $\mathrm{NE}_{00}^{1: 4} \mathrm{run}$, as in Fig. 15.
Lastly, in Fig. 20, we show the total recoil velocity along with the relative contributions from the dominant modes for the $\mathrm{NE}_{00}^{1: 4}$ run. Again, the qualitative behavior is quite similar to the $\mathrm{NE}_{00}^{2: 3}$ and $\mathrm{NE}_{00}^{1: 2}$ runs, but we can now identify a clear trend of a smaller anti-kick for smaller values of $\eta$. As mentioned above in Section VIC, the amplitude of the anti-kick is most strongly dependent on the relative amplitudes of the $S^{21}$ and $I^{33}$ modes, but for non-spinning BH binaries, these modes both scale the same with mass ratio. However, the amplitude of the $I^{22}$ mode decreases with decreasing $\eta$, while the amplitude of $I^{44}$ increases with decreasing $\eta$, at least over the range considered here. Thus the amplitude of the $F^{33,44}$ flux increases relative to the $F^{22,33}$ flux for more extreme mass ratios. From Figs. 15 and 20, we see that the $F^{22,33}$ flux dominates the anti-kick, while the $F^{33,44}$ flux contributes almost nothing to it, so by increasing the relative amplitude of $F^{33,44}$, we have effectively decreased the size of the anti-kick.
[1] F. Pretorius, Phys. Rev. Lett. 95, 121101 (2005).

[2] M. Campanelli, C.O. Lousto, P. Marronetti, and Y. Zlochower, Phys. Rev. Lett. 96, 111101 (2006).

[3] J. Baker, J. Centrella, D. Choi, M. Koppitz, and J. van Meter, Phys. Rev. Lett. 96, 111102 (2006).

[4] U. Sperhake, Phys. Rev. D 76, 104015 (2007).

[5] J. González, U. Sperhake, B. Brügmann, M. Hannam, and S. Husa, Phys. Rev. Lett. 98, 091101 (2007).

[6] B. Szilagyi, D. Pollney, L. Rezzolla, J. Thornburg and J. Winicour, Class. Quantum Grav. 24, S275 (2007).

[7] F. Herrmann, I. Hinder, D. Shoemaker, and P. Laguna, Class. Quantum Grav. 24, S33 (2007).

[8] J.G. Baker, J. Centrella, D. Choi, M. Koppitz, J.R. van Meter, and M.C Miller, Astrophys. J 653, L93 (2006).

[9] J.A. Gonzalez, U. Sperhake, B. Brügmann, M. Hannam, and S. Husa, Phys.Rev.Lett.98,091101 (2007).

[10] F. Herrmann, I. Hinder, D. Shoemaker, P. Laguna, and R.A. Matzner, Astrophys. J 661, 430 (2007).

[11] M. Koppitz, D. Pollney, C. Reisswig, L. Rezzolla, J. Thornburg, P. Diener, and E. Schnetter, Phys. Rev. Lett. 99,041102 (2007).

[12] J.G. Baker, W.D. Boggs, J.Centrella, B.J. Kelly, S.T. McWilliams, M.C. Miller, and J.R. van Meter, Astrophys. J 668, 1140 (2007).

[13] J.A. Gonzalez, M.D. Hannam, U. Sperhake, B. Brugmann, and S. Husa, Phys. Rev. Lett. 98, 231101 (2007).

[14] W. Tichy and P. Marronetti, Phys. Rev. D 76, 061502 (2007).

[15] B. Brügmann, J.A. Gonzalez, M. Hannam, S. Husa, and U. Sperhake, arXiv:0707.0135

[16] M. Campanelli, C.O. Lousto, Y. Zlochower, and D. Merritt, Astrophys. J. Lett. 659, L5 (2007).

[17] C.W. Misner, K.S. Thorne, and J.A. Wheeler, Gravitation, W.H. Freeman, San Francisco, 1973.

[18] J. Baker, M. Campanelli, C. Lousto, Phys. Rev. D 65, 044001 (2002).

[19] W.B. Bonnor and M.A. Rotenberg, Proc. R. Soc. London A 265, 109 (1961).
[20] A. Peres, Phys. Rev. 128, 2471 (1962).

[21] J.D. Bekenstein, Astrophys. J. 183, 657 (1973).

[22] F.I. Cooperstock, Astrophys. J. 213, 250 (1977).

[23] M.J. Fitchett, Mon. Not. R. Astr. Soc. 203, 1049 (1983); M.J. Fitchett and S. Detweiler, Mon. Not. R. Astr. Soc. 211, 933 (1984).

[24] M.G. Haehnelt, Mon. Not. R. Astr. Soc. 269, 199 (1994); K. Menou, Z. Haiman, and V.K. Narayanan, Astrophys.J. 558, 535 (2001); M. Volonteri, F. Haardt, and P. Madau, Astrophys.J. 582, 559 (2003).

[25] D. Merritt, M. Milosavljevic, M. Favata, and S.A. Hughes, Astrophys. J. 607, L9 (2004).

[26] M. Volonteri, Astrophys. J 663, L5 (2007).

[27] J.D. Schnittman, Astrophys. J 667, L133 (2007).

[28] M.G. Haehnelt, M.B. Davies, and M.J. Rees, Mon. Not. R. Astr. Soc. 366, L22 (2006).

[29] E.W. Bonning and G.A. Shields, Astrophys. J 666, L13 (2007).

[30] M. Boylan-Kolchin, C.-P. Ma, and E. Quataert, Astrophys. J. Lett. 613, L37 (2004).

[31] T. R. Lauer, et al., Astrophys. J 662, 808 (2007).

[32] J.D. Schnittman and A. Buonanno, Astrophys.J. 662, L63 (2007).

[33] J.D. Schnittman, Phys. Rev. D 70, 124020 (2004).

[34] T. Bogdanovic, C.S. Reynolds, and M.C. Miller, Astroph. J 661, L147 (2007).

[35] A. Loeb, Phys. Rev. Lett. 99, 041103 (2007).

[36] See, e.g., L. Blanchet, Living Rev. Rel. 5, 3 (2002).

[37] T. Damour, B.R. Iyer and B.S. Sathyaprakash, Phys. Rev. D 57, 885 (1998).

[38] A. Buonanno and T. Damour, Phys. Rev. D 59, 084006 (1999).

[39] A. Buonanno and T. Damour, Phys. Rev. D 62, 064015 (2000).

[40] T. Damour, P. Jaranowski, and G. Schäfer, Phys. Rev. D 62, 084011 (2000).

[41] T. Damour, P. Jaranowski, and G. Schäfer, Phys. Rev. D 62, 044024 (2000). 
[42] A. Buonanno, Y. Chen, and T. Damour, Phys. Rev. D 74, 104005 (2006).

[43] A. Wiseman, Phys. Rev. D 46, 1517 (1992).

[44] L. Kidder, Phys. Rev. D 52, 821 (1995).

[45] M. Favata et al., Astrophys. J. 607, L5 (2004).

[46] L. Blanchet, M.S.S. Qusailah, and C.M. Will, Astrophys. J. 635, 508 (2006).

[47] T. Damour and A. Gopakumar, Phys. Rev. D 73, 124006 (2006).

[48] C.F. Sopuerta, N. Yunes, and P. Laguna, Astrophys. J. Lett. 656, L9 (2007).

[49] R.H. Price and J. Pullin, Phys. Rev. Lett. 72, 3297 (1994).

[50] K.S. Thorne, Rev. Mod. Phys. 52, 299 (1980).

[51] L. Blanchet and T. Damour, Ann. Inst. H. Poincaré 50, 377 (1989).

[52] L. Blanchet and G. Schäfer, Mon. Not. R. Astr. Soc. 239, 845 (1989).

[53] L. Blanchet, T. Damour, and G. Schäfer, Mon. Not. R. Astr. Soc. 242, 289 (1990).

[54] W. Junker and G. Schäfer, Mon. Not. R. Astr. Soc. 254, 146 (1992).

[55] C.V. Vishveshwara, Nature 227, 936 (1970); M. Davis, R. Ruffini, W.H. Press and R.H. Price, Phys. Rev. Lett. 27, 1466 (1971); W. Press, Astrophys J. Letters 170, L105 (1971); M. Davis, R. Ruffini and J. Tiomno, Phys. Rev. D 5, 2932 (1972); S. Chandrasekhar and S. Detweiler, Proc. R. Soc. Lond. A 344, 441 (1975).

[56] J.N. Goldberg, A.J. Macfarlane, E.T. Newman, F. Rohrlich, and E.C.G. Sundarshan, J. Math. Phys. 8, 2155 (1967).

[57] Y. Wiaux, L. Jacques, P. Vandergheynst, astro-ph/0508514.

[58] S. Brandt and B. Brügmann, Phys. Rev. Lett. 78, 3606 (1997).

[59] J.D. Brown and L.L. Lowe, J. Comput. Phys. 209, 582 (2005).
[60] J. Bowen and J.W. York, Phys. Rev. D 21, 2047 (1980).

[61] D. Christodoulou, Phys. Rev. Lett. 25, 1596 (1970); D. Christodoulou and R. Ruffini, Phys. Rev. D 4, 3552 (1971).

[62] B. Imbiriba, J.G. Baker, D.-I. Choi, J. Centrella, D.R. Fiske, J.D. Brown, J.R. van Meter, and K. Olson, Phys. Rev. D 70, 124025 (2004).

[63] P. Hübner, Class. Quantum Grav. 16, 2823 (1999).

[64] M.D. Duez, S.L. Shapiro, and H.-J. Yo, Phys. Rev. D 69, 104016 (2004).

[65] J. van Meter, J.G. Baker, M. Koppitz, and D.-I. Choi, Phys. Rev D 73, 124011 (2006).

[66] J.G. Baker and J. van Meter, Phys. Rev. D 72, 104010 (2005).

[67] P. MacNeice, K. Olson, C. Mobarry, R. de Fainchtein, and C. Packer, Computer Physics Comm. 126, 330 (2000).

[68] J.G. Baker, S.T. McWilliams, J.R. van Meter, J. Centrella, D.I. Choi, B.J. Kelly, and M. Koppitz, Phys. Rev. D 75, 124024 (2007).

[69] K. Martel and E. Poisson, Phys. Rev. D 71, 104003 (2005).

[70] E.W. Leaver, Proc. R. Soc. Lond. A 402, 285 (1985).

[71] F. Echeverria, Phys. Rev. D 40, 3194 (1997).

[72] E. Berti, V. Cardoso and C. Will, Phys. Rev. D 73, 064030 (2006).

[73] A. Buonanno, G. Cook and F. Pretorius, Phys. Rev. D 75, 124018 (2007).

[74] L. Blanchet and B. Iyer, Class. Quant. Grav. 20, 755 (2003).

[75] E. Berti et al., Phys. Rev. D 76, 064034 (2007).

[76] L. Blanchet, A. Buonanno, and G. Faye, Phys. Rev. D 74, 104034 (2006); Erratum-ibid. D 75, 049903 (2007).

[77] A. Buonanno, Y. Pan, J.G. Baker, J. Centrella, B.J. Kelly, S.T. McWilliams, and J.R. van Meter, arXiv:0706.3732 